Supporting Information

\title{
Metal-Organic Framework (MOF) Derived Recyclable, Superhydrophobic Composite of Cotton Fabrics for the Facile Removal of Oil Spills
}

\author{
Rana Dalapati, Soutick Nandi, Chiranjib Gogoi, Arpita Shome and Shyam Biswas*
}

Department of Chemistry, Indian Institute of Technology Guwahati, Guwahati, 781039 Assam, India

* To whom correspondence should be addressed. E-mail: sbiswas@iitg.ac.in; Tel: 913612583309 . 
Movie S1: Hydrophobicity of SH-UiO-66@CFs

Movie S2: Absorption-based selective oil/water separation of heavy oils by SH-UiO-66@CFs Movie S3: Absorption-based selective oil/water separation of light oils by SH-UiO-66@CFs Movie S4: Filtration-based selective oil/water separation by SH-UiO-66@CFs Movie S5: Collection of the underwater oil against gravity by SH-UiO-66@CFs

Materials and Physical Measurements. All the reagent grade starting materials and solvents were used without further purification as received from commercial suppliers. Perkin Elmer Spectrum Two FT-IR spectrometer was used to record Fourier transform infrared spectra in the range of 440-4000 $\mathrm{cm}^{-1}$. To describe the FT-IR absorption bands, the following standard classifications were used: weak (w), very strong (vs), strong (s), medium (m), broad (br) and shoulder (sh). A Bruker D2 Phaser X-ray diffractometer working at $30 \mathrm{kV}, 10 \mathrm{~mA}$ was employed for the collection of ambient temperature X-Ray powder diffraction (XRPD) patterns using $\mathrm{Cu}-\mathrm{K} \alpha(\lambda=1.5406 \AA)$ radiation. A Mettler-Toledo TGA/SDTA 851e thermogravimetric analyzer was used for conducting the thermogravimetric analyses in the temperature range of $25-700{ }^{\circ} \mathrm{C}$ under air atmosphere with a heating rate of $5{ }^{\circ} \mathrm{C} \mathrm{min}^{-1}$. Quantachrome Autosorb iQMP gas sorption analyser was utilized for the nitrogen sorption experiments at $-196{ }^{\circ} \mathrm{C}$ up to 1 bar. A Bruker Avance III 600 spectrometer was utilized for recording ${ }^{1} \mathrm{H}-\mathrm{NMR}$ at $600 \mathrm{MHz}$. The mass spectrum (in ESI mode) was measured with an Agilent 6520 Q-TOF high-resolution mass spectrometer Contact angle experiments were performed by employing a KRUSS Drop Shape Analyzer-DSA25 instrument with an automatic liquid dispenser at ambient temperature. Field emission - scanning electron microscopy (FE-SEM) images were collected by Zeiss (Zemini) scanning electron microscope. Cotton was purchased from local medical shop of Guwahati city (Assam, India). Kerosene oil and gasoline were purchased from a local shop of Guwahati city (Assam, India). Digital images were captured using a Nikon Coolpix B500 digital camera.

Synthesis of 2-Trifluoroacetamidoterephthalic Acid $\left(\mathbf{H}_{2} \mathbf{L}\right)$ Ligand. In a $50 \mathrm{~mL}$ round bottom flask, $1.81 \mathrm{~g}(10 \mathrm{mmol})$ of 2-aminoterephthalic acid was added slowly in $15 \mathrm{~mL}$ of trifluoroacetic anhydride at room temperature and the reaction mixture was stirred for $4 \mathrm{~h}$. After that the reaction mixture was slowly poured in $20 \mathrm{~mL}$ of ice-cold water and a white precipitate appeared. The obtained white solid product of 2-trifluoroacetamidoterephthalic acid $\left(\mathrm{H}_{2} \mathbf{L}\right)$ was filtered and washed with large amount of water. Yield: $69 \%(1.89 \mathrm{~g}, 6.9 \mathrm{mmol}) .{ }^{1} \mathrm{H}-\mathrm{NMR}: \delta$ $12.57(\mathrm{~s}, 1 \mathrm{H}), 8.78(\mathrm{~s}, 1 \mathrm{H}), 8.13(\mathrm{~d}, 1 \mathrm{H}), 7.88(\mathrm{~d}, 1 \mathrm{H}) \mathrm{ppm} ;{ }^{13} \mathrm{C}$ NMR: $\delta 169.14,166.48,155.25$, 137.85, 135.87, 131.99, 126.31, 123.44, 122.38, 116.92, 115.00 ppm; ${ }^{19}$ F NMR: $\delta-75.20$ ppm. ESI-MS (m/z): 300.0140 for $(\mathrm{M}+\mathrm{Na})^{+}$ion $\left(\mathrm{M}=\right.$ mass $\mathrm{H}_{2} \mathbf{L}$ ligand). The NMR and mass spectra of $\mathrm{H}_{2} \mathbf{L}$ ligand are shown in Figures S1-S4 (Supporting Information).

Absorption Capacities for Various Oils by SH-UiO-66@CFs Composite. In the oil absorption test, pre-weighed ( 10-15 mg) hydrophobic SH-UiO-66@CFs composite was placed in $10 \mathrm{~mL}$ of various heavy oils (dichloromethane, chloroform and carbon tetrachloride) and light oils (ethyl acetate, toluene, hexane, motor oil, silicone oil, gasoline and kerosene) for 
1 min to reach absorption equilibrium and then removed and weighed. All the experiments were made at room temperature. The absorption capacity (wt $\%)$ is defined as:

Absorption capacity $(\mathrm{wt} \%)=\left(\mathrm{W}_{4}-\mathrm{W}_{3}\right) / \mathrm{W}_{3} \times 100 \%$

The absorption capacity in $(\mathrm{g} / \mathrm{g})$ unit is defined as:

Absorption capacity $(\mathrm{g} / \mathrm{g})=\mathrm{W}_{4} / \mathrm{W}_{3}$

where, $\mathrm{W}_{3}$ is weight of oven-dried SH-UiO-66@CFs and $\mathrm{W}_{4}$ is the weight of oil-absorbed SH-UiO-66@CFs. At least 10 measurements were carried out for each oil sample and the average value was plotted.

Absorption Based Separation of Oil and Water by SH-UiO-66@CFs Composites. For the separation of light oil from oil/water mixture, single piece of SH-UiO-66@CFs ( 100-120 $\mathrm{mg}$ ) was exposed to different oil/water mixtures that composed of $5 \mathrm{~mL}$ of oil and $40 \mathrm{~mL}$ of water. The oil phase was immediately soaked in SH-UiO-66@CFs and the separated oil was collected by squeezing the material manually. On the other hand, for the separation of heavy oil from oil/water mixture, a piece of SH-UiO-66@CFs $(\sim 100-120 \mathrm{mg})$ was brought into contact with the sediment oil. The modified cotton selectively absorbed the oil phase after coming into contact with the oil phase. All the experiments were performed at room temperature. The separation efficiency (\%) is defined as:

Separation efficiency $(\%)=\mathrm{V}_{2} / \mathrm{V}_{1} \times 100 \%$

where, $\mathrm{V}_{1}$ is the volume of oil $(\mathrm{mL})$ used and $\mathrm{V}_{2}$ is the absorbed volume of oil ( $\mathrm{mL}$ ) by SHUiO-66@CFs. Ten measurements were carried out for each oil sample and the average value was plotted.

Absorption Based Separation of Oil and Water by SH-UiO-66@CFs Composite under Different Conditions. This oil/water absorption-based separation process was further extended with several complex aqueous phases, such as tap water, lake water, river water, artificial sea water and water having different $\mathrm{pH}$ (2 and 12). Tap water was collected from IIT Guwahati campus, water of IIT Guwahati lake was used as lake water, water of Brahmaputra river (Assam, India) was utilized as river water. The artificial sea water was prepared by mixing $\mathrm{MgSO}_{4}(0.325 \mathrm{~g}), \mathrm{MgCl}_{2}(0.226 \mathrm{~g}), \mathrm{CaCl}_{2}(0.112 \mathrm{~g})$ and $\left.\mathrm{NaCl}(2.673 \mathrm{~g})\right)$ in $100 \mathrm{~mL}$ deionized water in a volumetric flask. Motor oil was used as model oil for this experiment.

Reusability of SH-UiO-66@CFs. Mechanical squeezing by finger was used to extract the absorbed oil from the SH-UiO-66@CFs. The absorbed recovered oil was used again without any post-treatment. After collecting the oil from SH-UiO-66@CFs, the material was washed with THF to remove rest of the oil and heated in air oven at $80^{\circ} \mathrm{C}$ for $2 \mathrm{~h}$. The oven dried SHUiO-66@CFs material was used further for the next set of oil absorption experiments. 


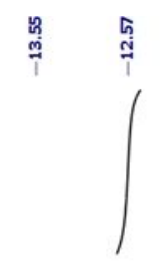

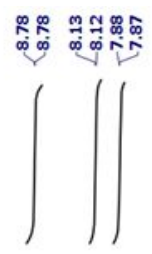

$\mathrm{COOH}_{\mathrm{O}}^{\mathrm{COOH}}$

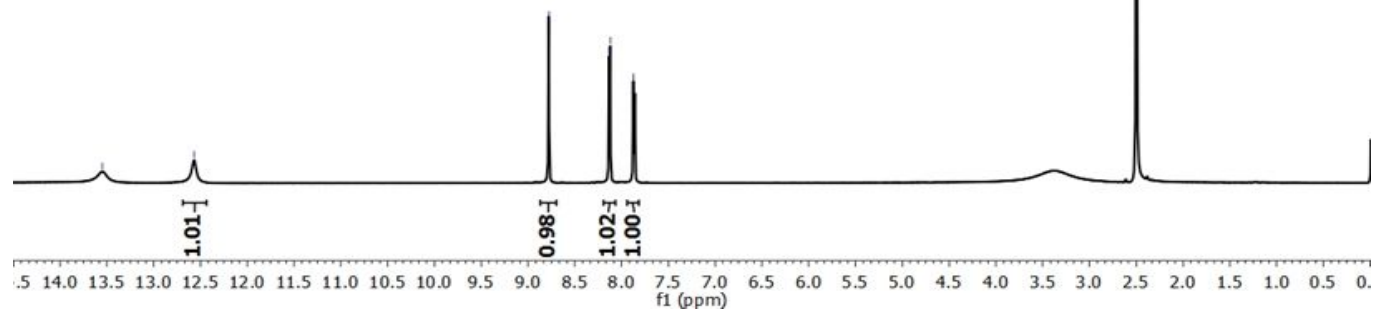

Figure S1. ${ }^{1} \mathrm{H}$ NMR spectrum of $\mathrm{H}_{2} \mathbf{L}$ ligand measured in DMSO- $d_{6}$.

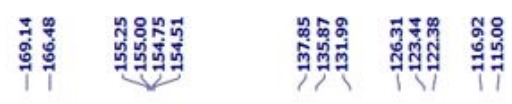<smiles>O=C(O)c1ccc(C(=O)O)c(NC(=O)C(F)(F)F)c1</smiles>

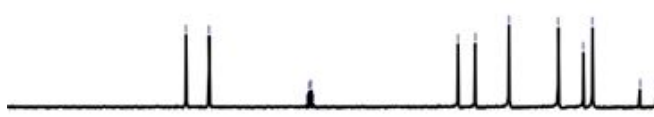

$\begin{array}{llllllll}180 & 170 & 160 & 150 & 140 & 130 & 120 & 110_{\mathrm{f} 1(\mathrm{ppm})}{ }^{100}\end{array}$

Figure S2. ${ }^{13} \mathrm{C}$ NMR spectrum of $\mathrm{H}_{2} \mathbf{L}$ ligand measured in DMSO- $d_{6}$. 
<smiles>O=C(O)c1ccc(C(=O)O)c(NC(=O)C(F)(F)F)c1</smiles>

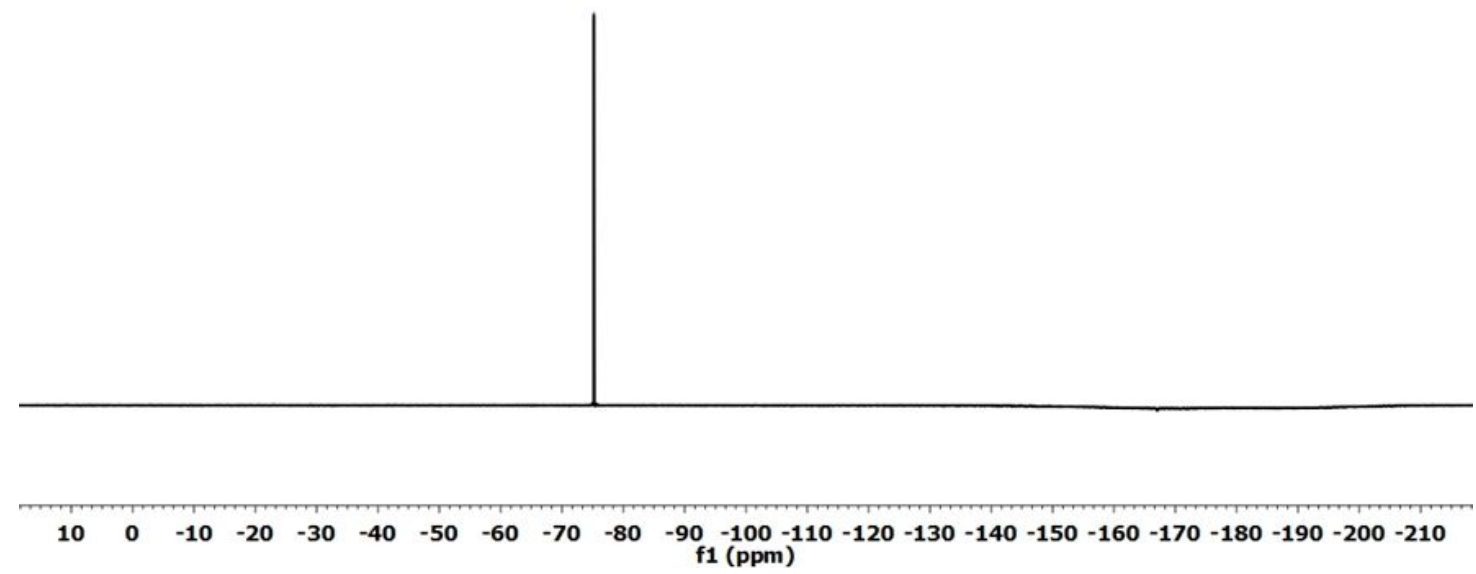

Figure S3. ${ }^{19} \mathrm{~F}$ NMR spectrum of $\mathrm{H}_{2} \mathbf{L}$ ligand measured in DMSO- $d_{6}$.

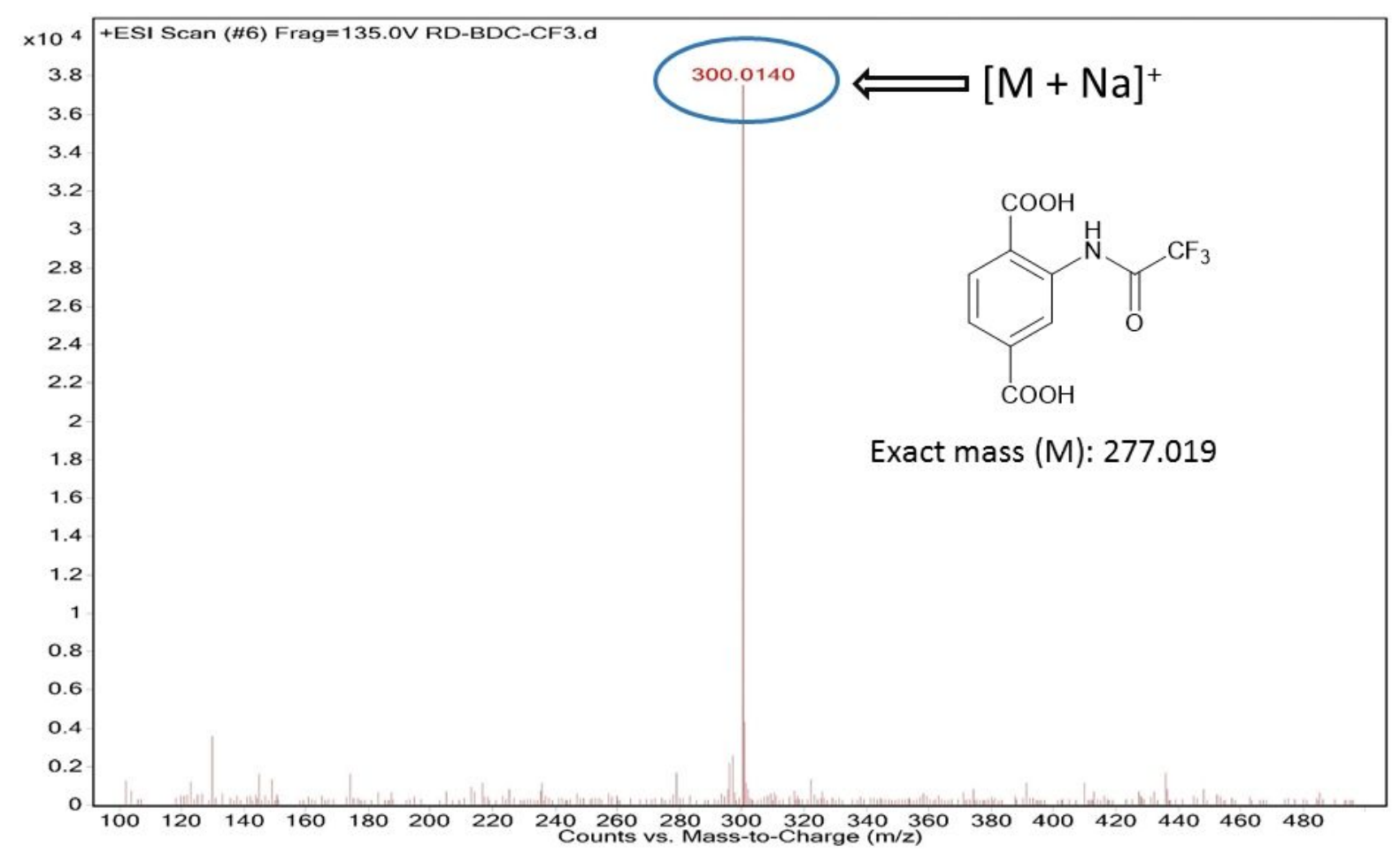

Figure S4. ESI-MS spectrum of $\mathrm{H}_{2} \mathbf{L}$ ligand $(\mathrm{M}+\mathrm{Na})^{+}$measured in methanol. 


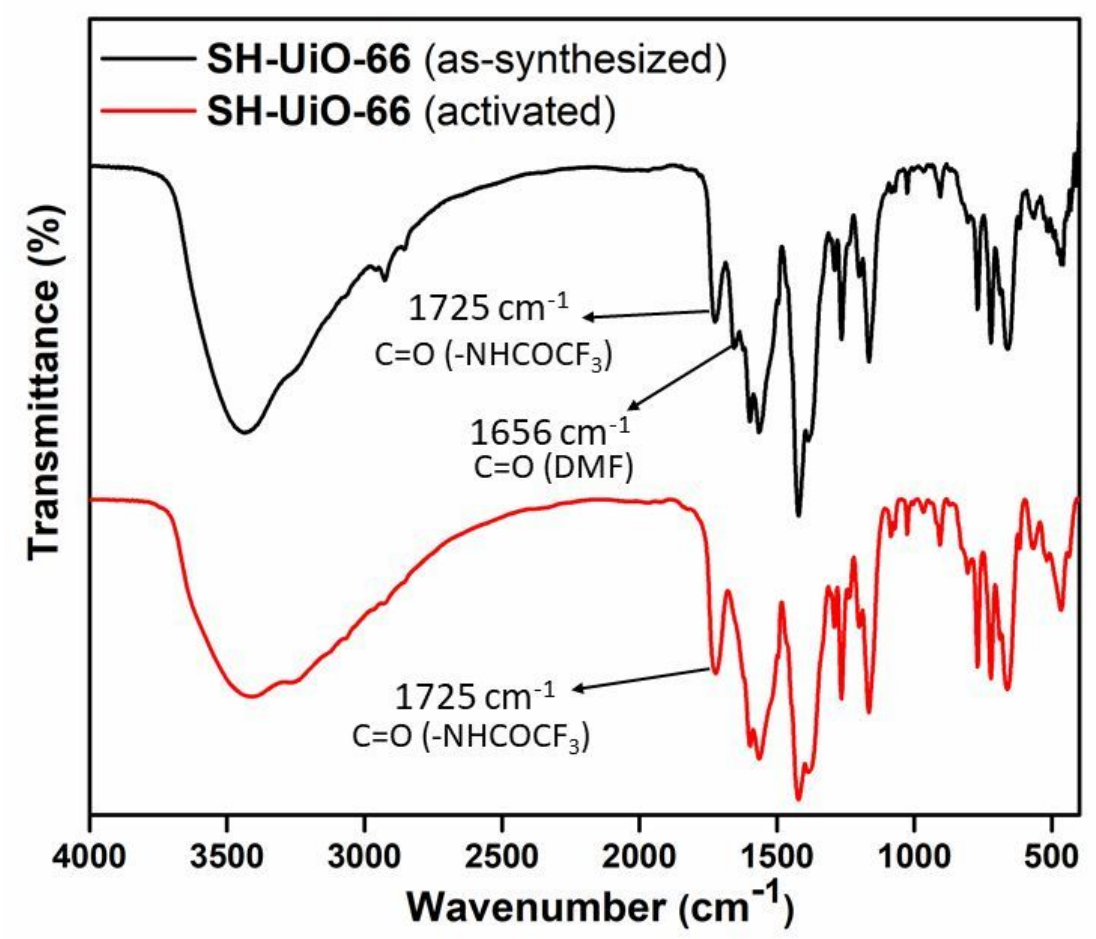

Figure S5. FT-IR spectra of as-synthesized (black) and thermally activated (red) form of SHUiO-66.

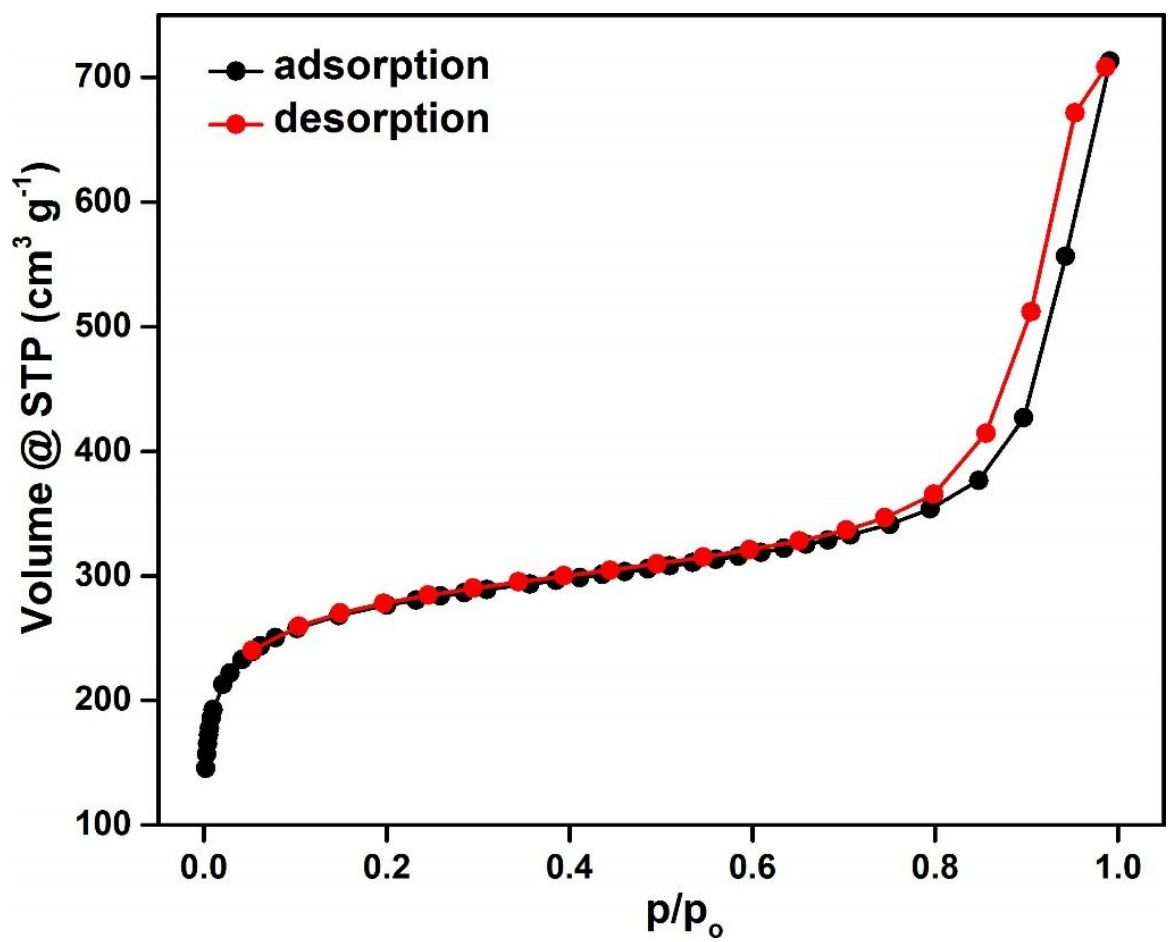

Figure S6. $\mathrm{N}_{2}$ adsorption (black circles) and desorption (red circles) isotherms of activated SH-UiO-66 recorded at $-196{ }^{\circ} \mathrm{C}$. 


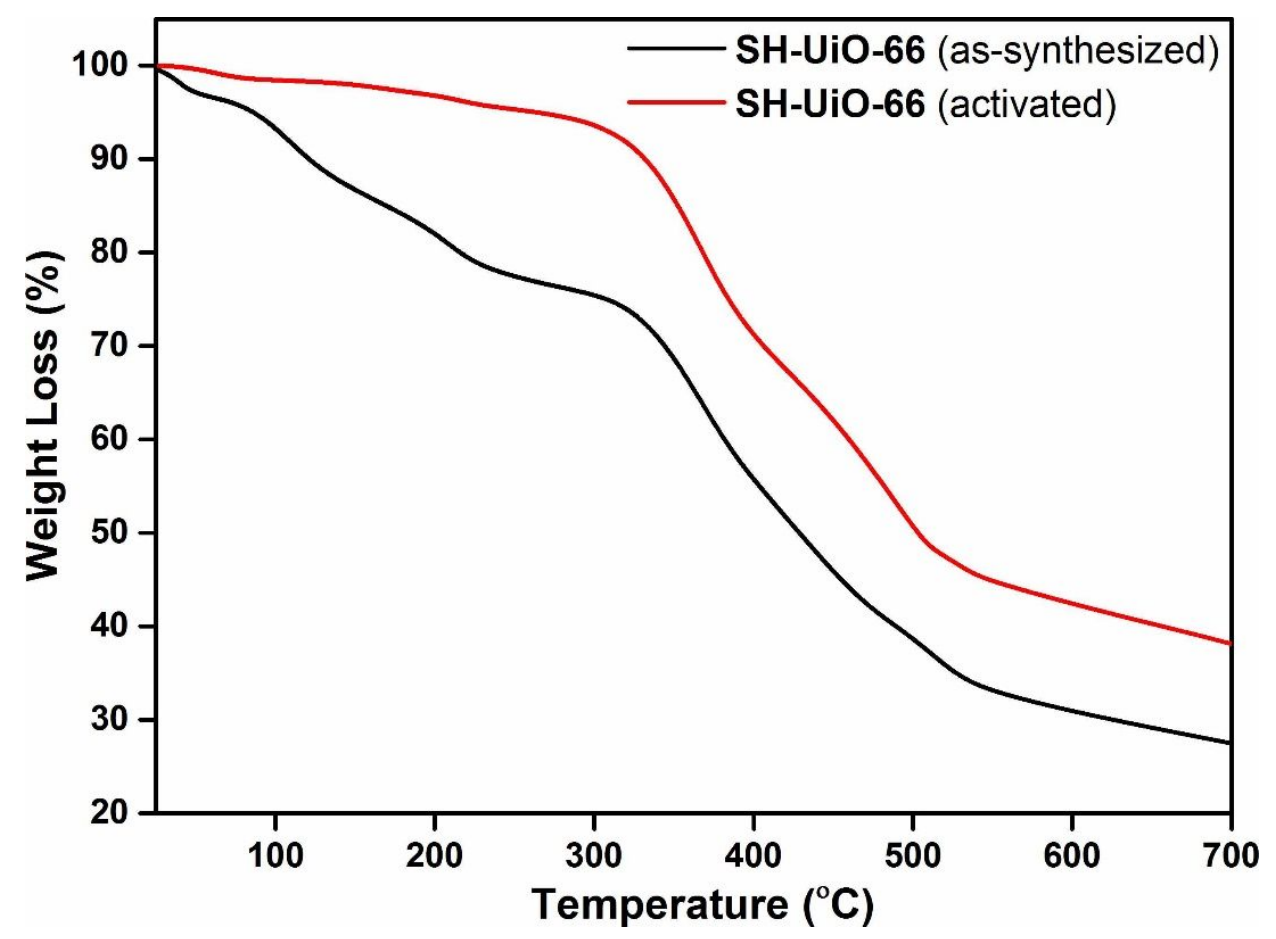

Figure S7. TG curves of as-synthesized and activated SH-UiO-66 recorded in an air atmosphere in the temperature range of $25-700{ }^{\circ} \mathrm{C}$ with a heating rate of $5{ }^{\circ} \mathrm{C} \mathrm{min}^{-1}$.

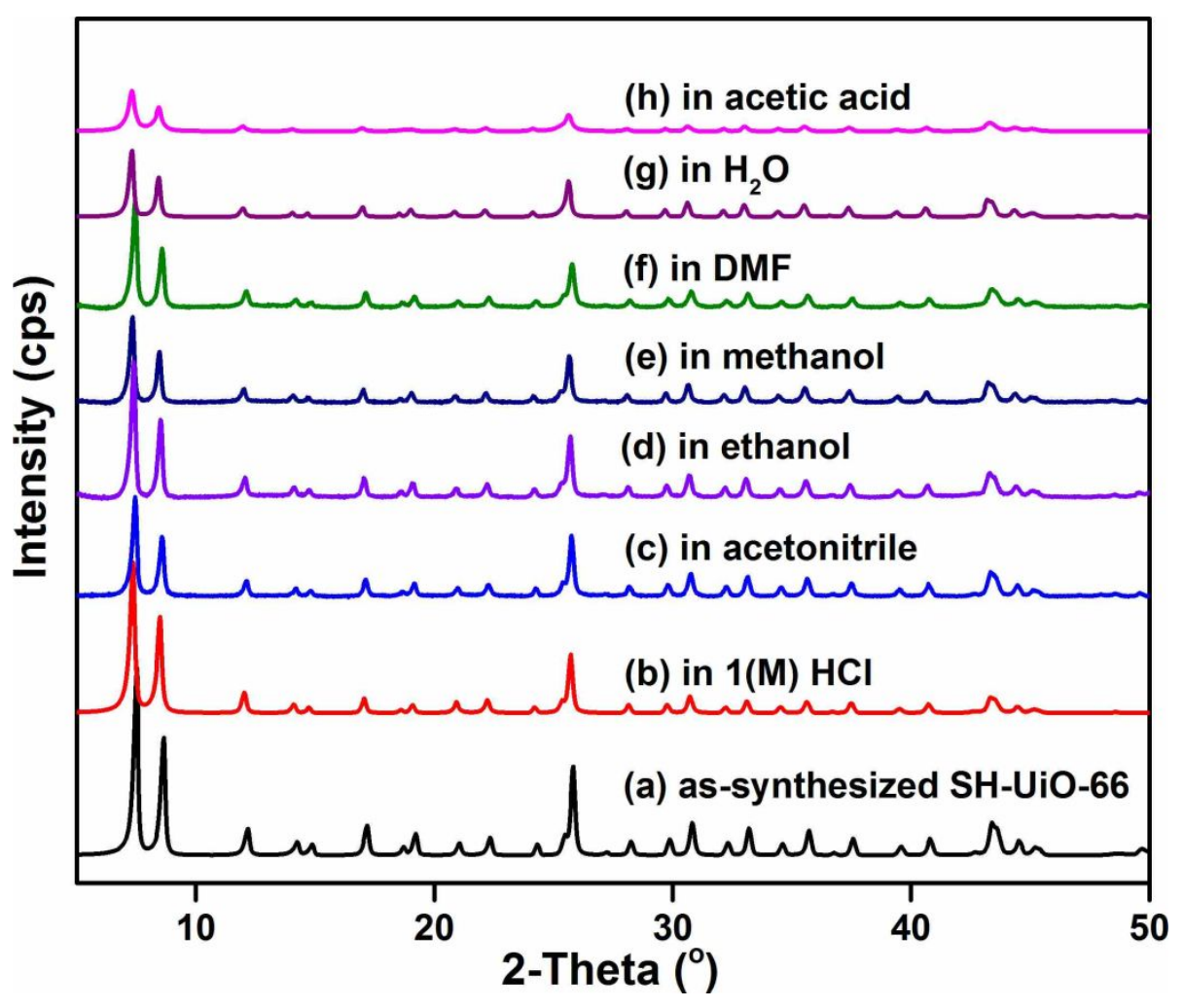

Figure S8. XRPD patterns of as-synthesized SH-UiO-66 (a), in 1M HCl (b), in acetonitrile (c), in ethanol (d) in methanol (e), in DMF (f), in water (g) and in acetic acid (h). 


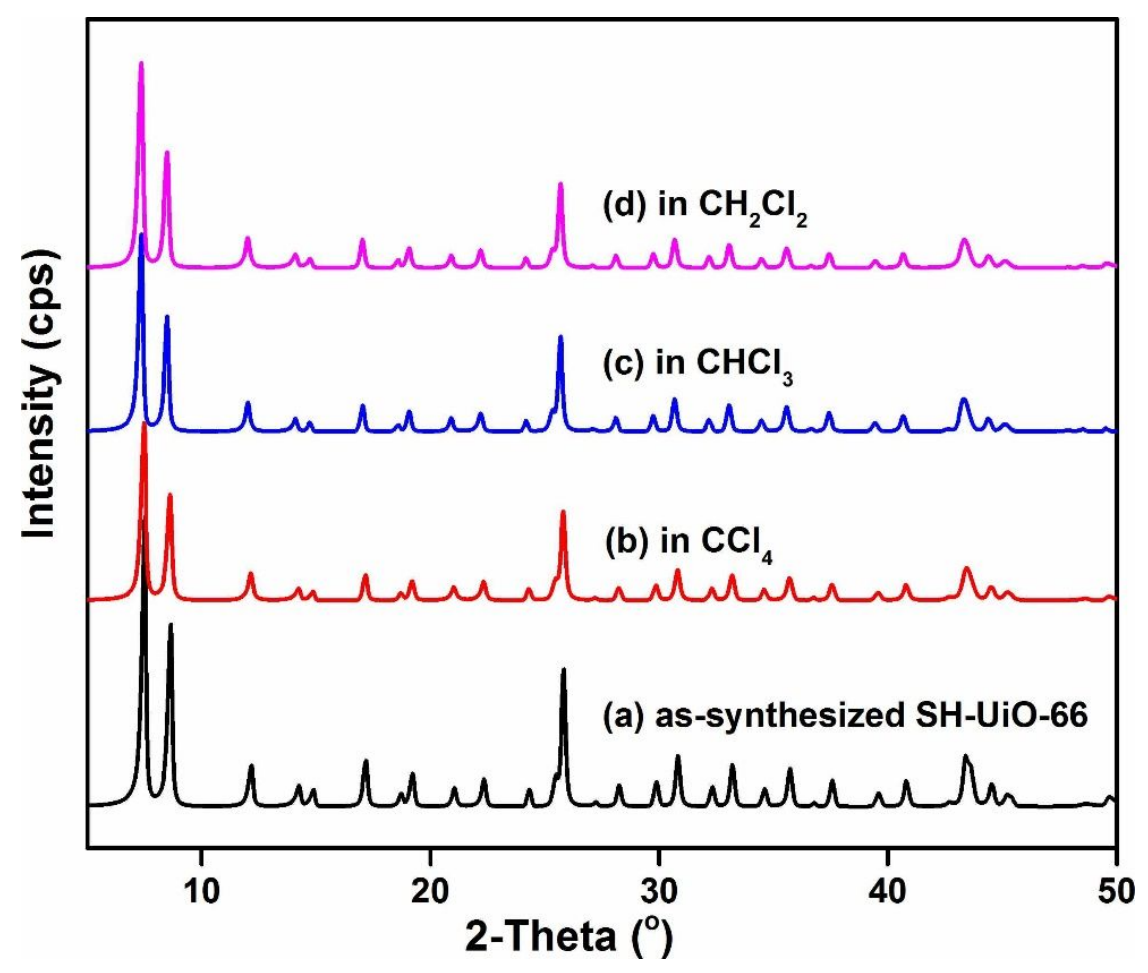

Figure S9. XRPD patterns of SH-UiO-66 in different heavy oils.

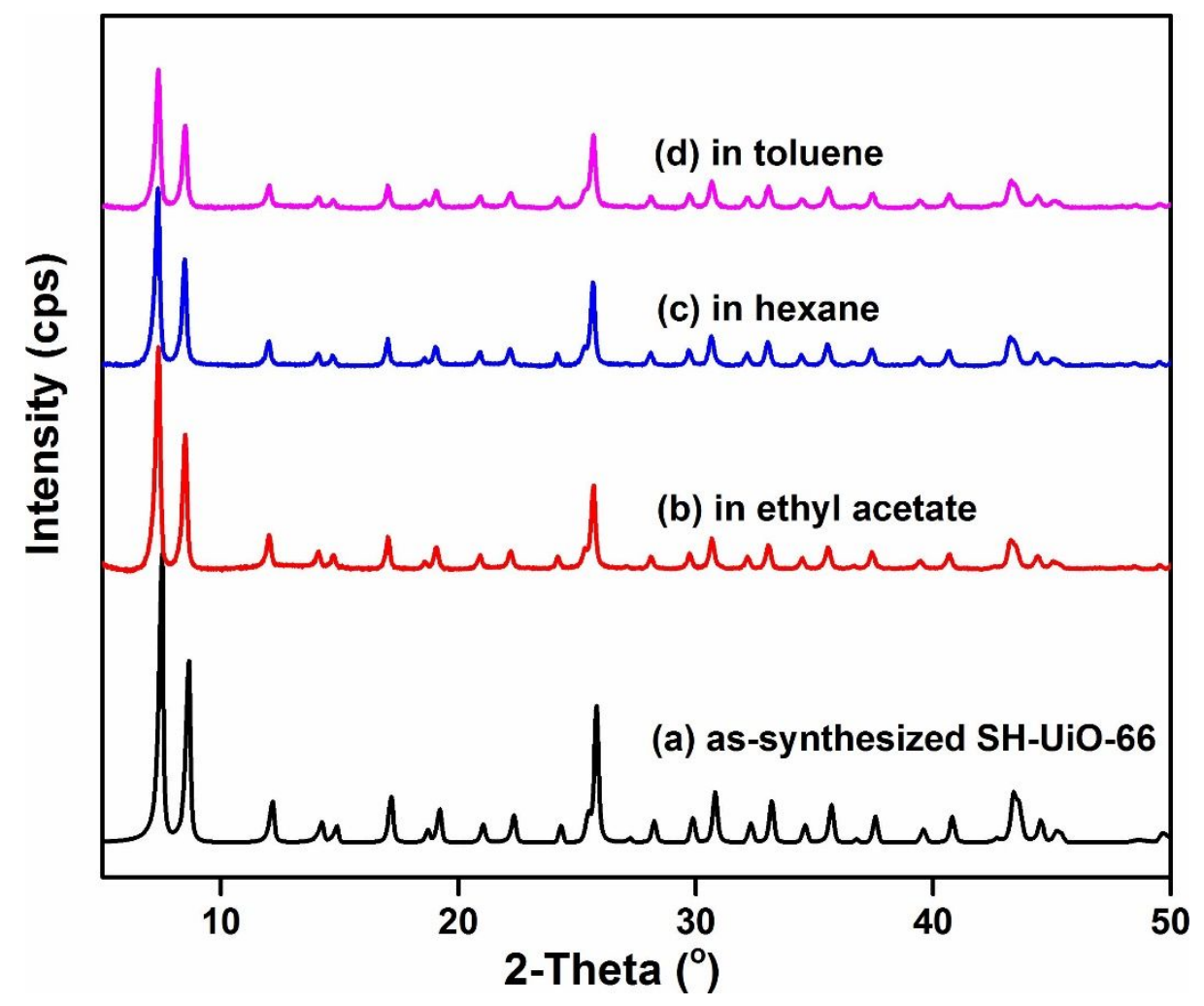

Figure S10. XRPD patterns of SH-UiO-66 in different light oils. 


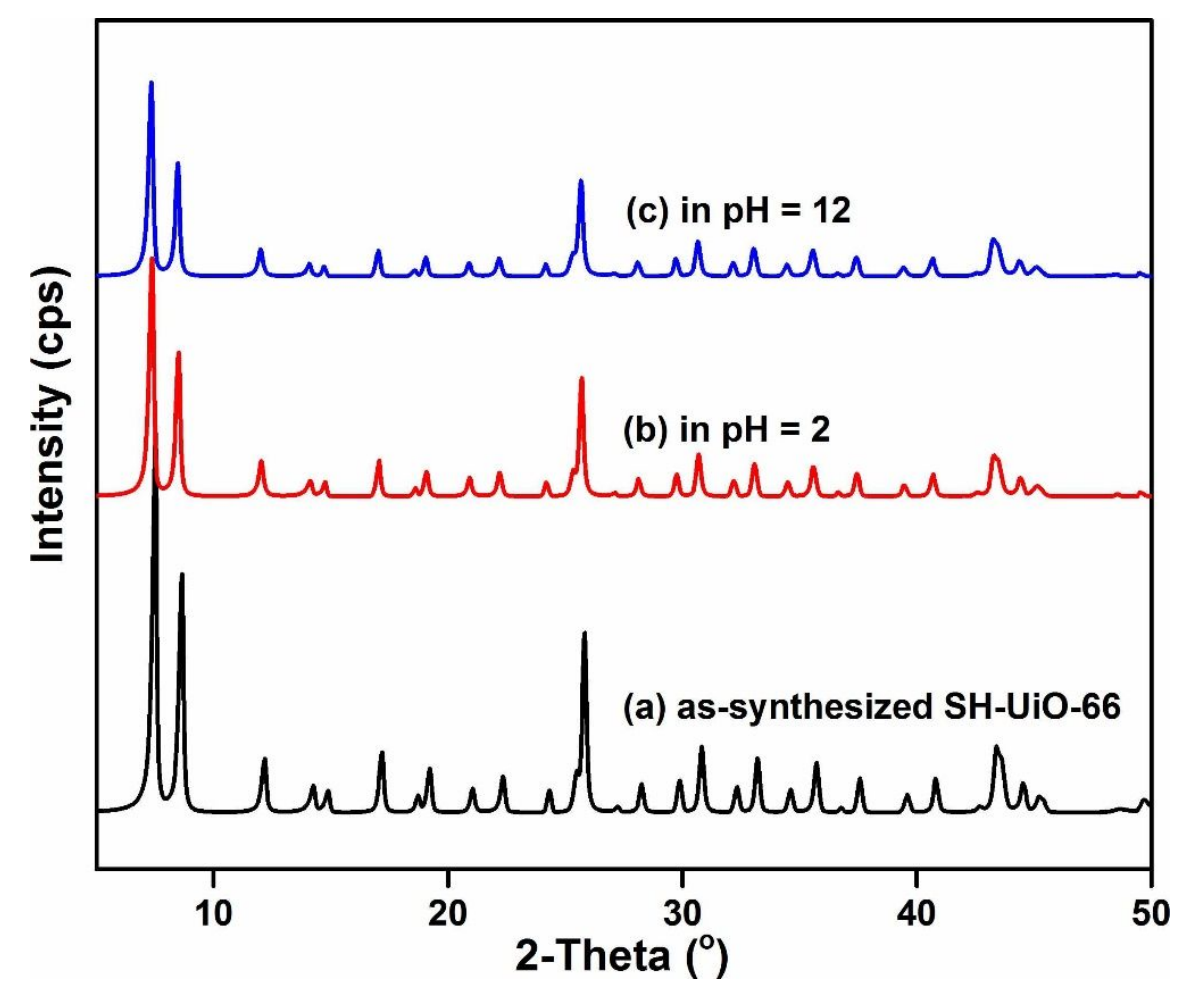

Figure S11. Aqua stability of SH-UiO-66 in different $\mathrm{pH}$ media.
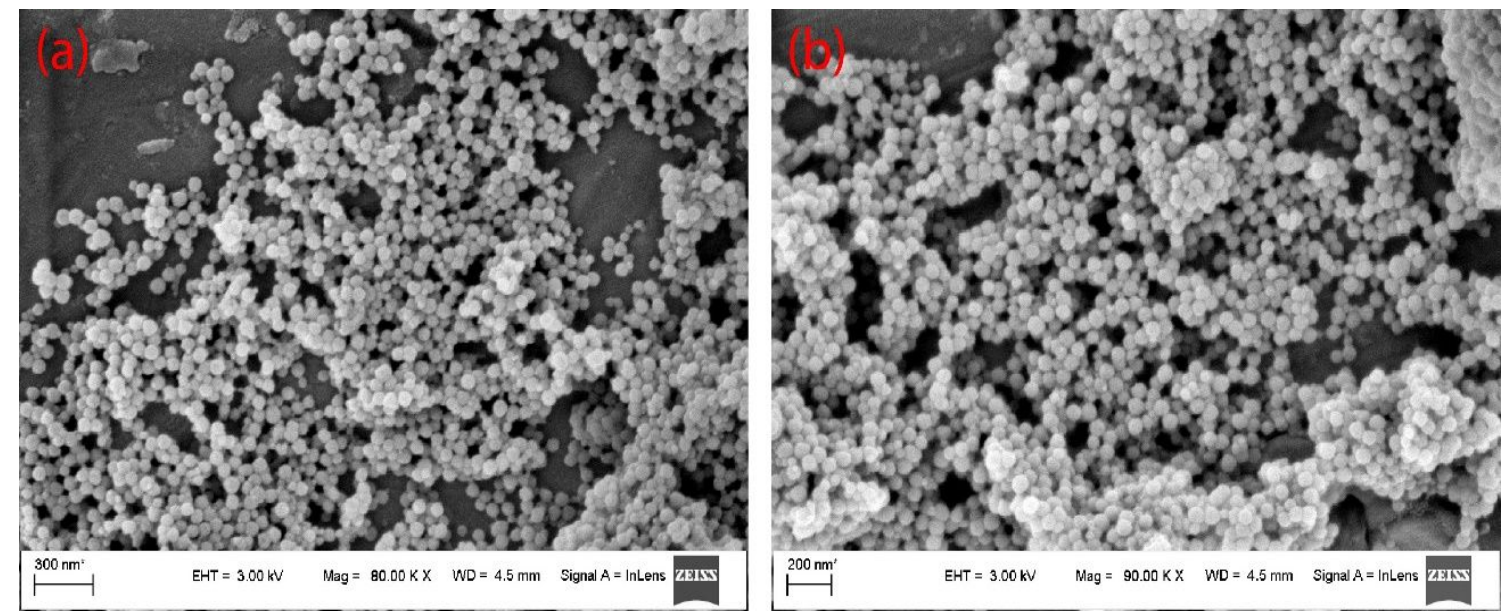

Figure S12. FE-SEM image of SH-UiO-66 showing a homogeneous phase of monodispersed spherical particles, which suggest the phase purity of the material. 


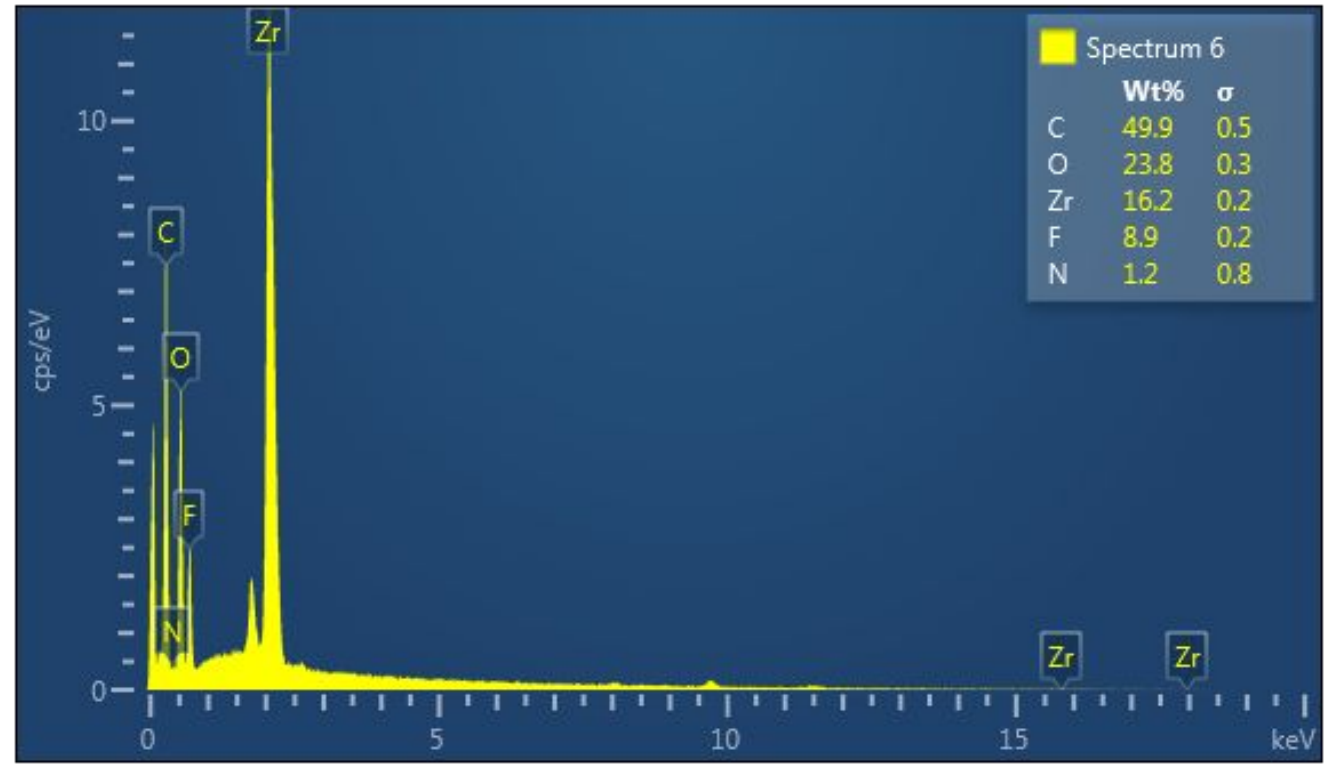

Figure S13. EDX spectrum of SH-UiO-66.

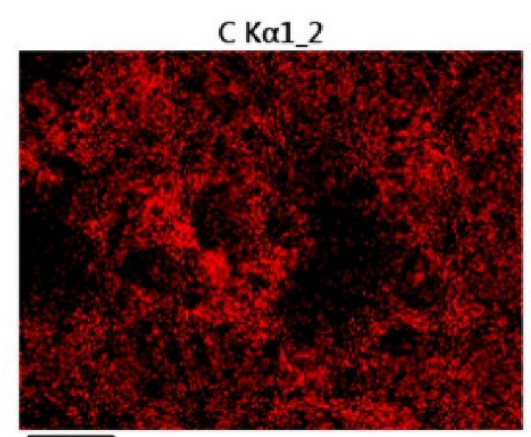

$\widehat{100 \mu \mathrm{m}}$
$\operatorname{Zr} \operatorname{L} \alpha 1$

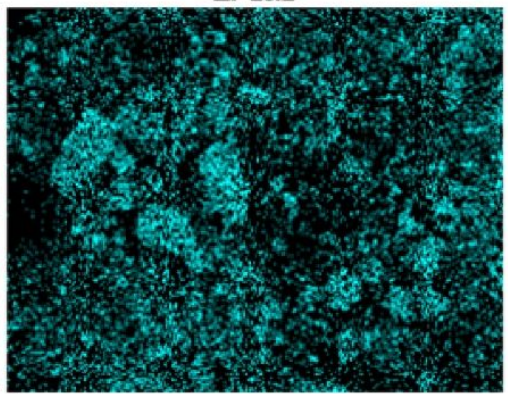

$\longdiv { 1 0 0 \mu \mathrm { m } }$

F K $\alpha 12$

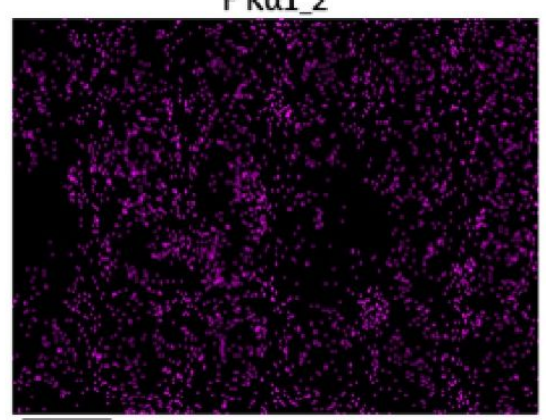

$\longdiv { 1 0 0 \mu \mathrm { m } }$
$\mathrm{O} K \alpha 1$

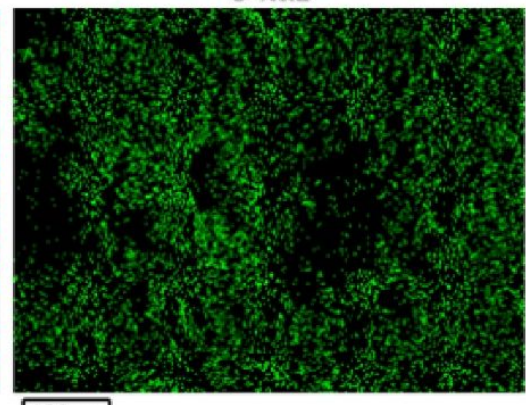

$\widehat{100 \mu \mathrm{m}}$

N Ka1_2

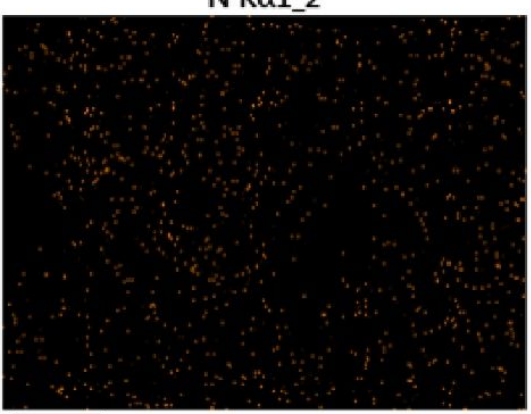

$\widehat{100 \mu \mathrm{m}}$

Figure S14. EDX elemental mapping of SH-UiO-66. 


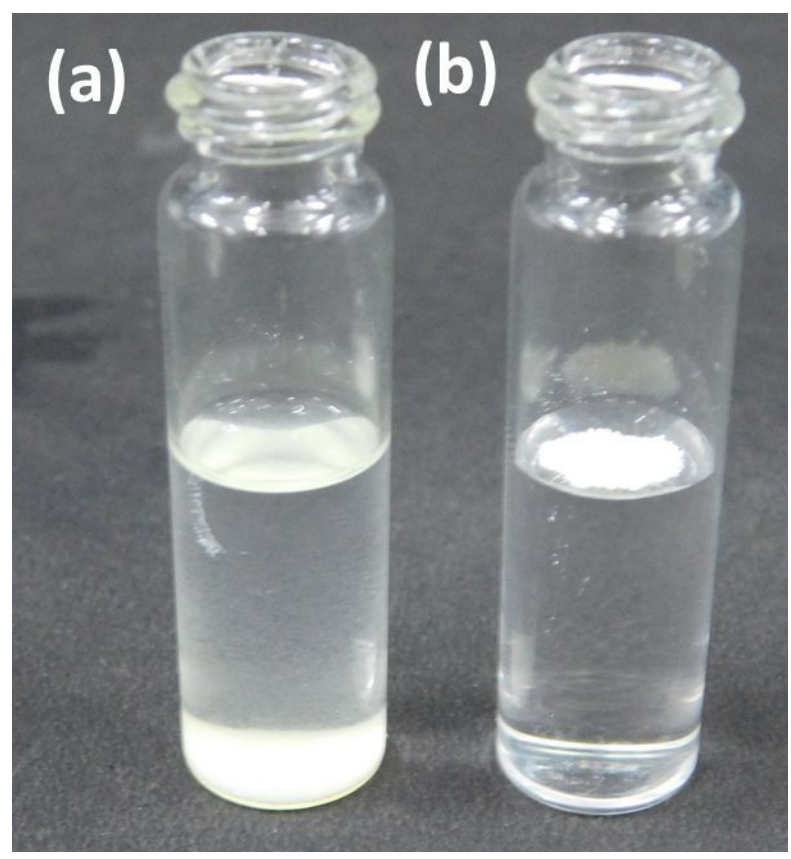

Figure S15. Self-floating ability of SH-UiO-66: in hexane (a) and in water (b).

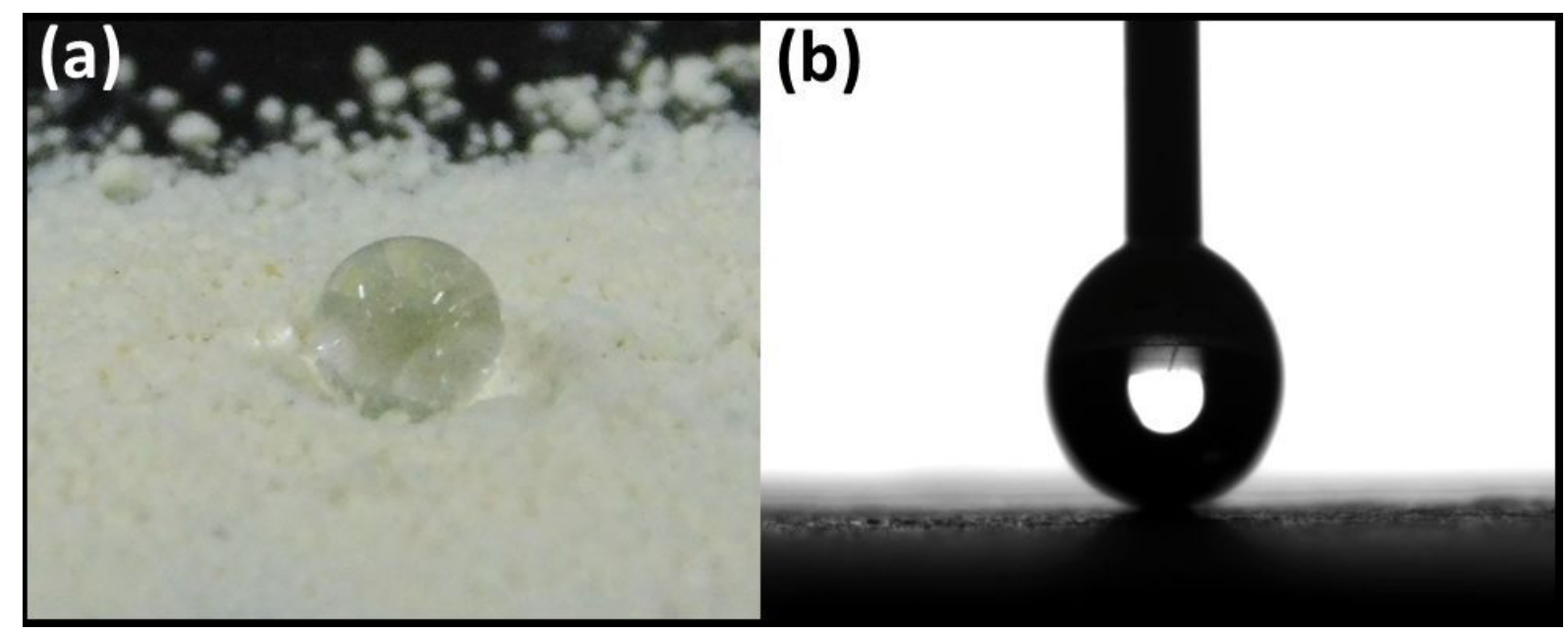

Figure S16. Water droplet suspended on SH-UiO-66 MOF: naked eye proof of hydrophobicity (a); Image of a water droplet slowly cast on the hydrophobic surface of the SH-UiO-66 MOF pellet with a contact angle of about $160^{\circ}$ (b). 


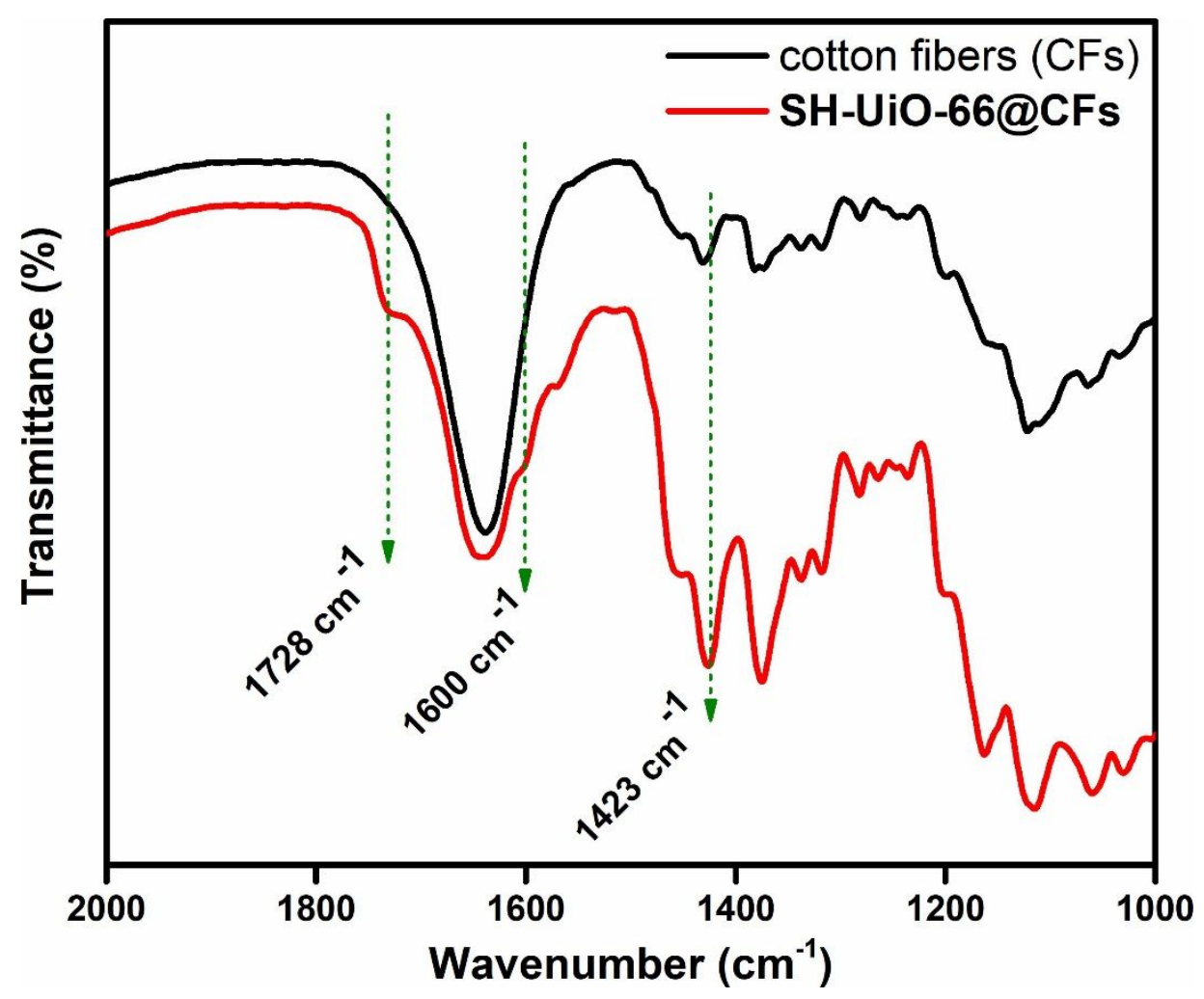

Figure S17. FT-IR spectra of cotton fibers and SH-UiO-66@CFs composite.

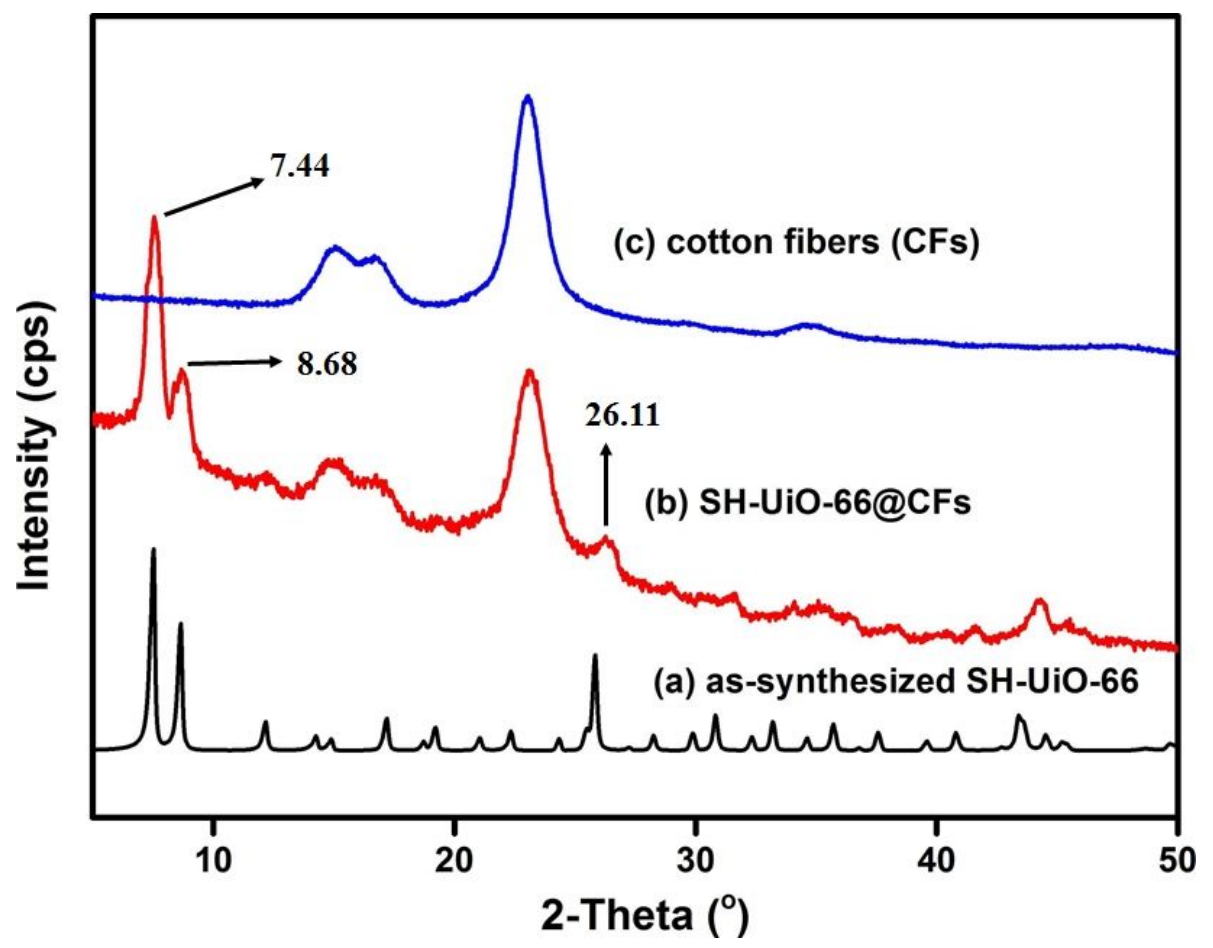

Figure S18. XRPD patterns of as-synthesized SH-UiO-66, SH-UiO-66@@CFs (b) and cotton fibers (c). 


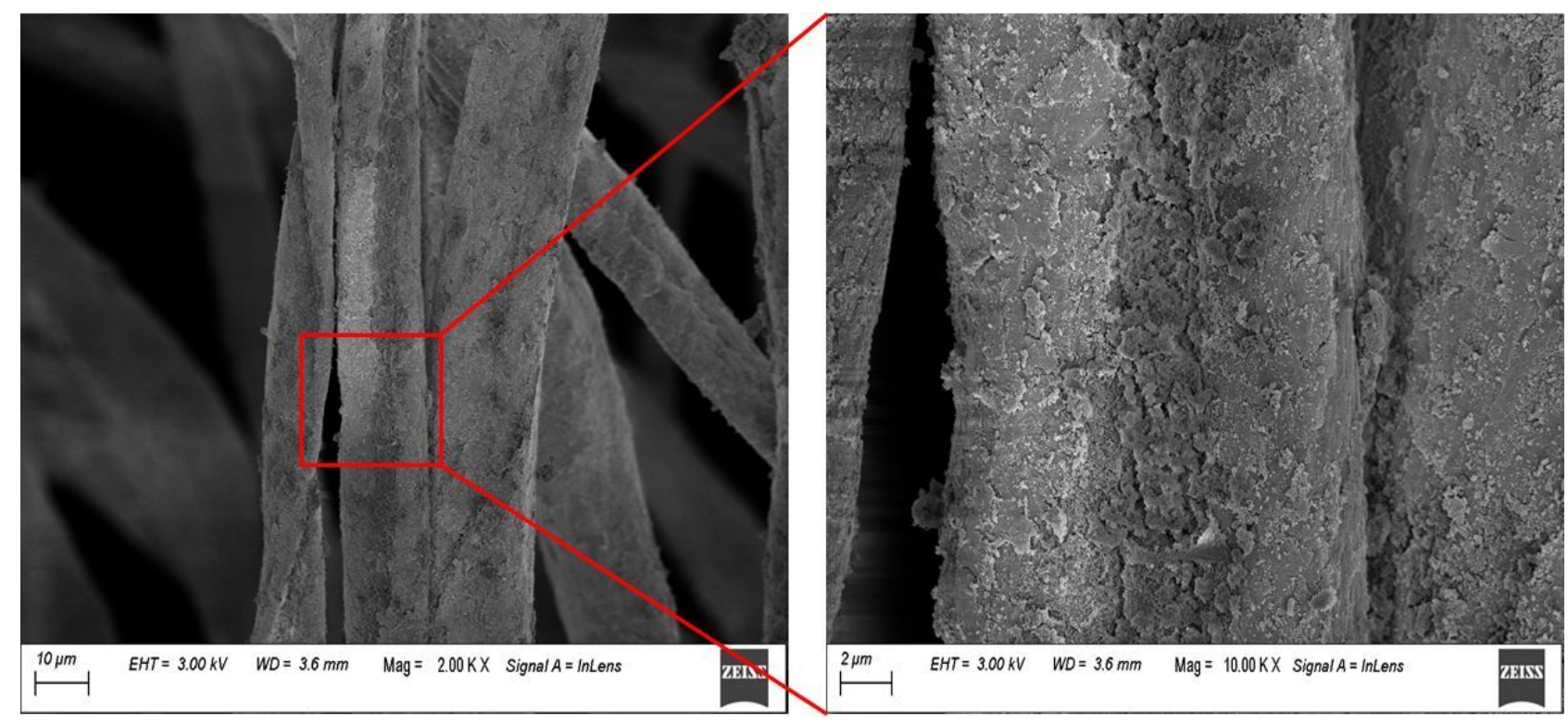

Figure S19. High resolution FE-SEM images of SH-UiO-66@CFs.

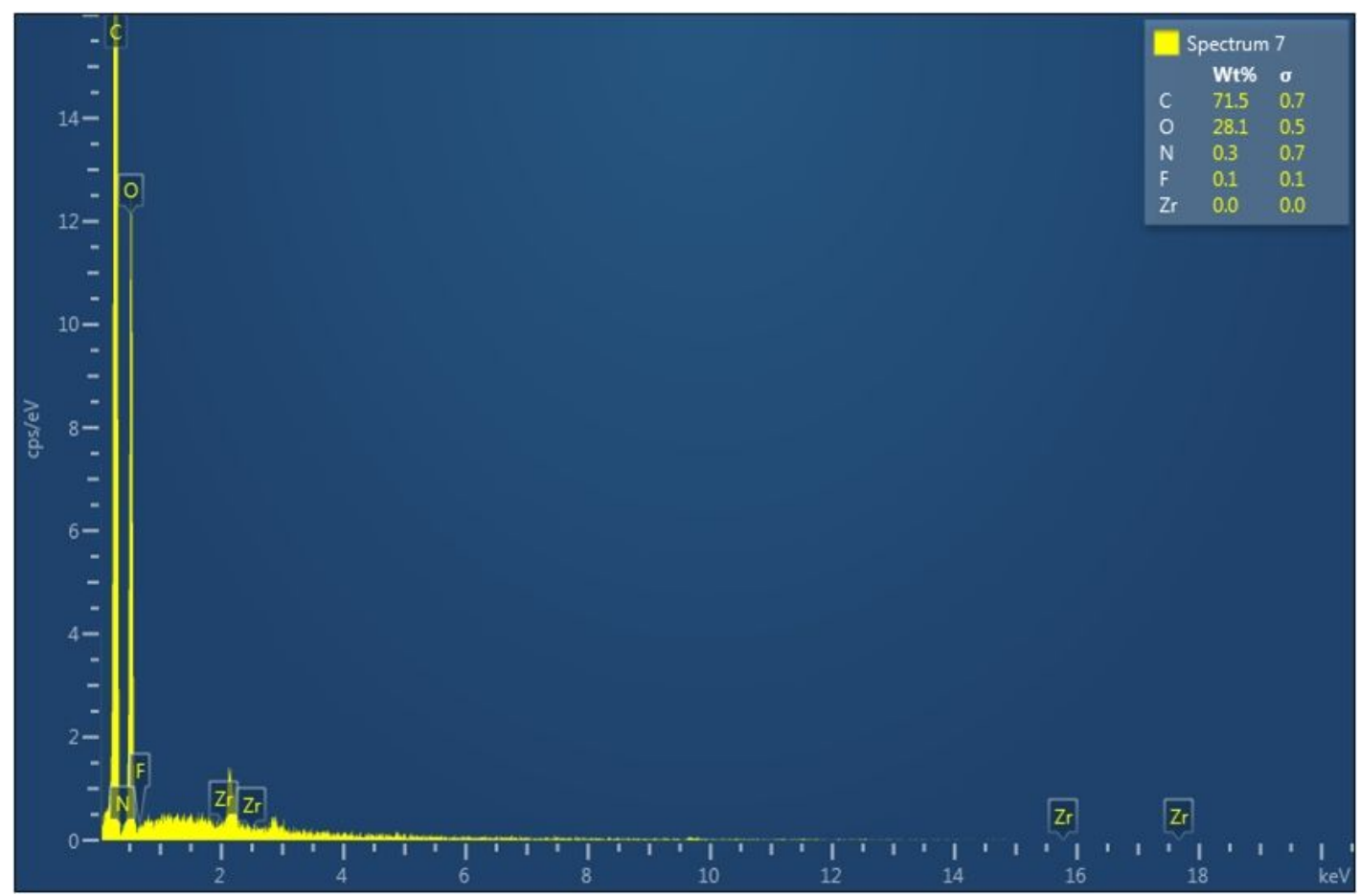

Figure S20. EDX spectrum of native cotton fiber. 


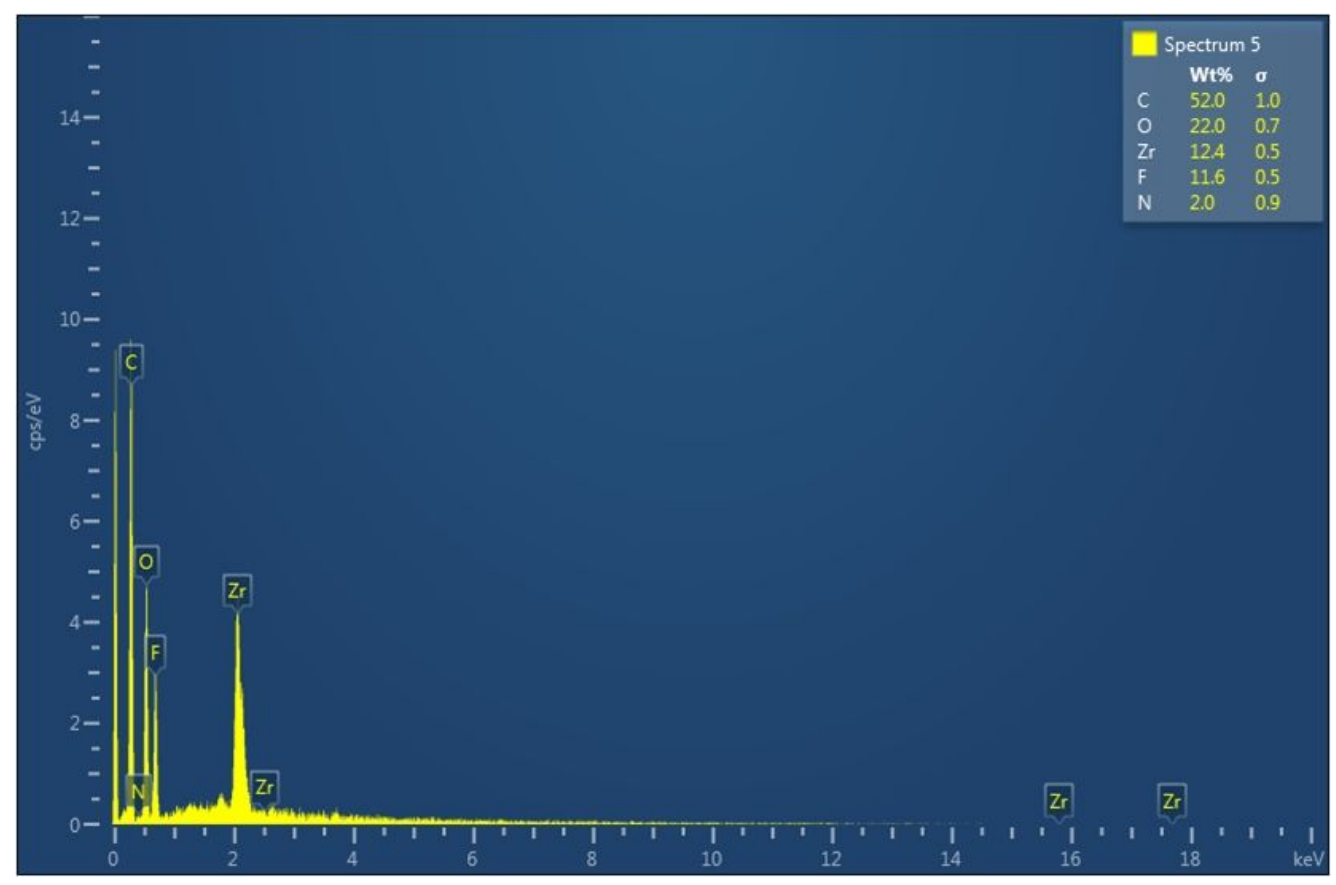

Figure S21. EDX spectrum of SH-UiO-66@CFs.
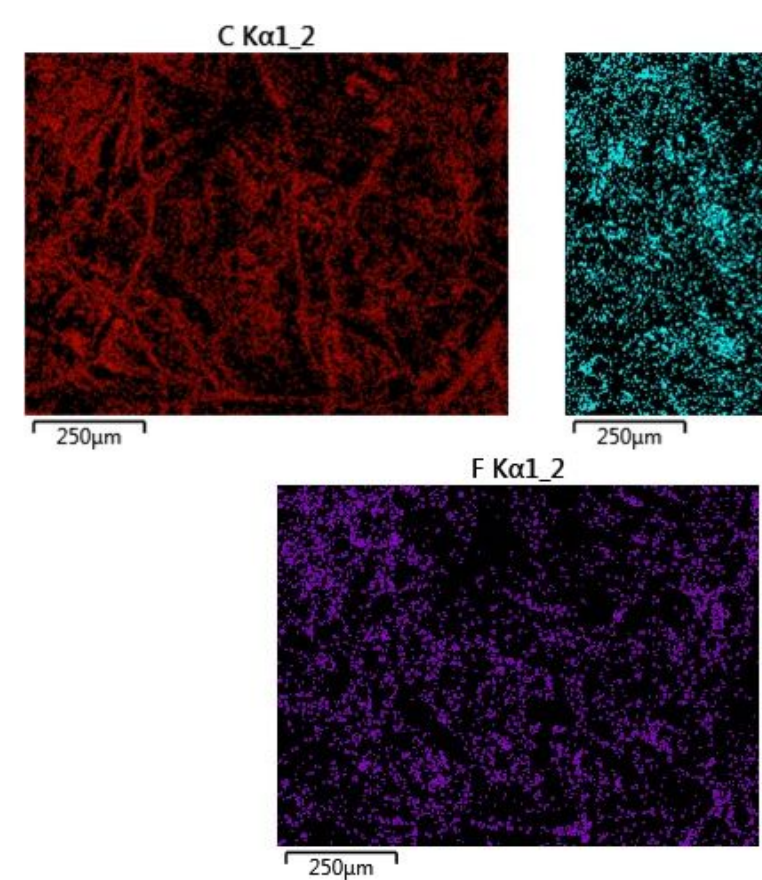

$\mathrm{Zr} \mathrm{L \alpha 1}$

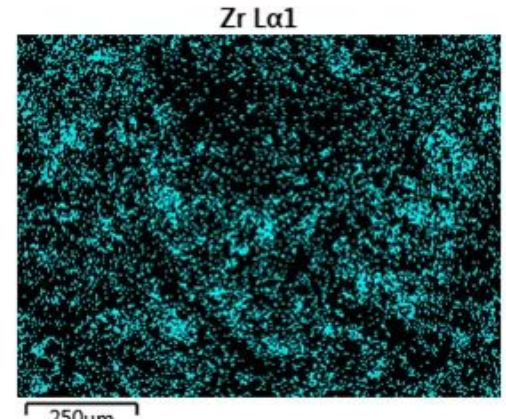

N Ka1_2
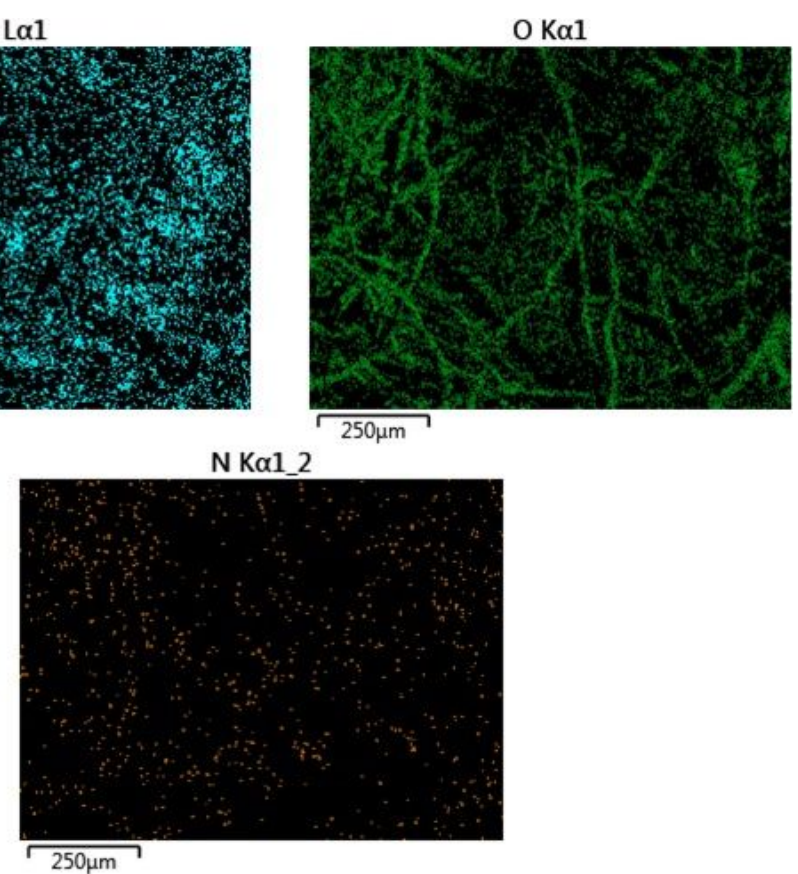

Figure S22. EDX elemental mapping of SH-UiO-66@CFs. 


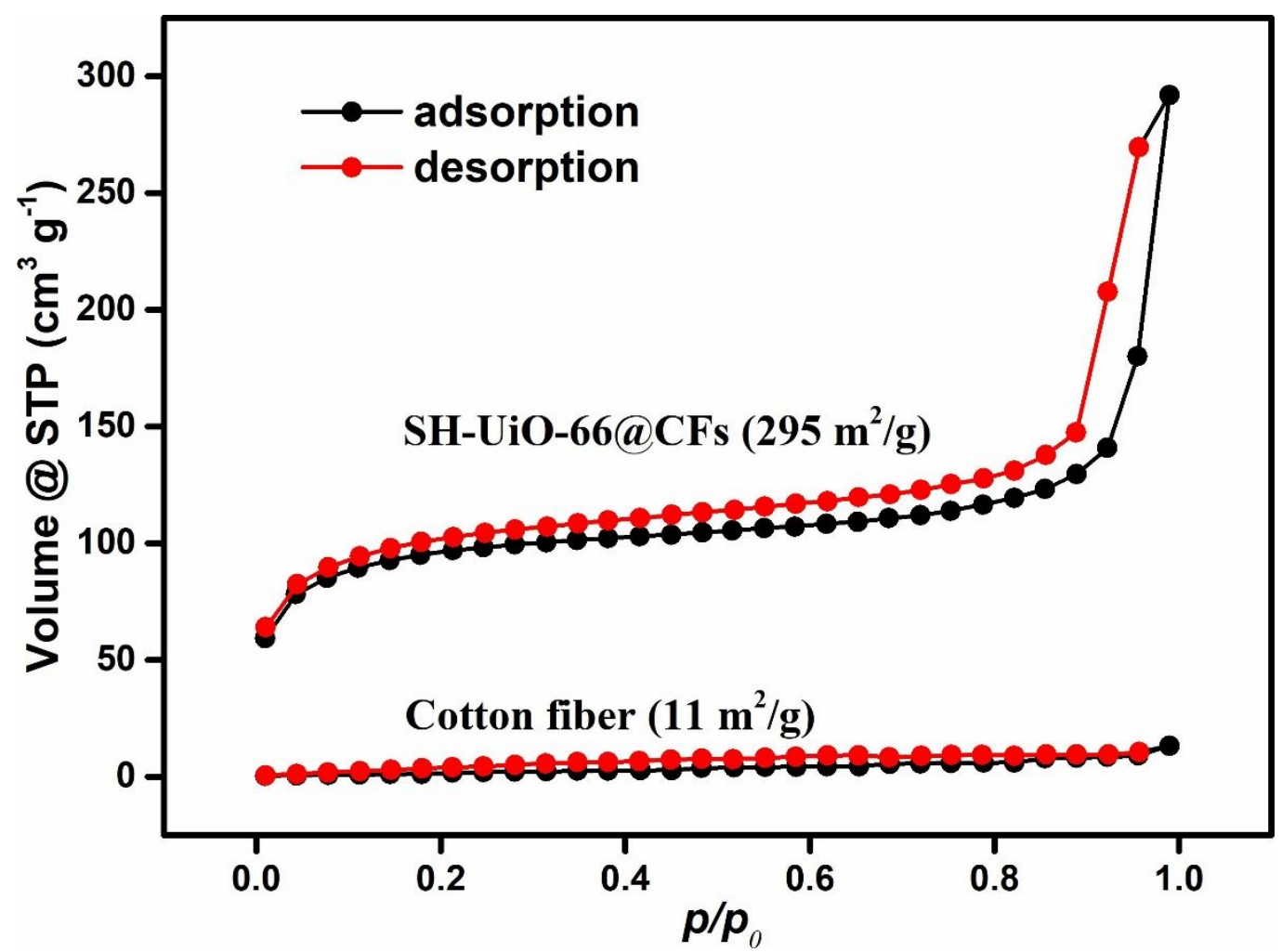

Figure S23. $\mathrm{N}_{2}$ adsorption (black circles) and desorption (red circles) isotherms of SH-UiO66@CFs and native cotton fiber recorded at $-196^{\circ} \mathrm{C}$.

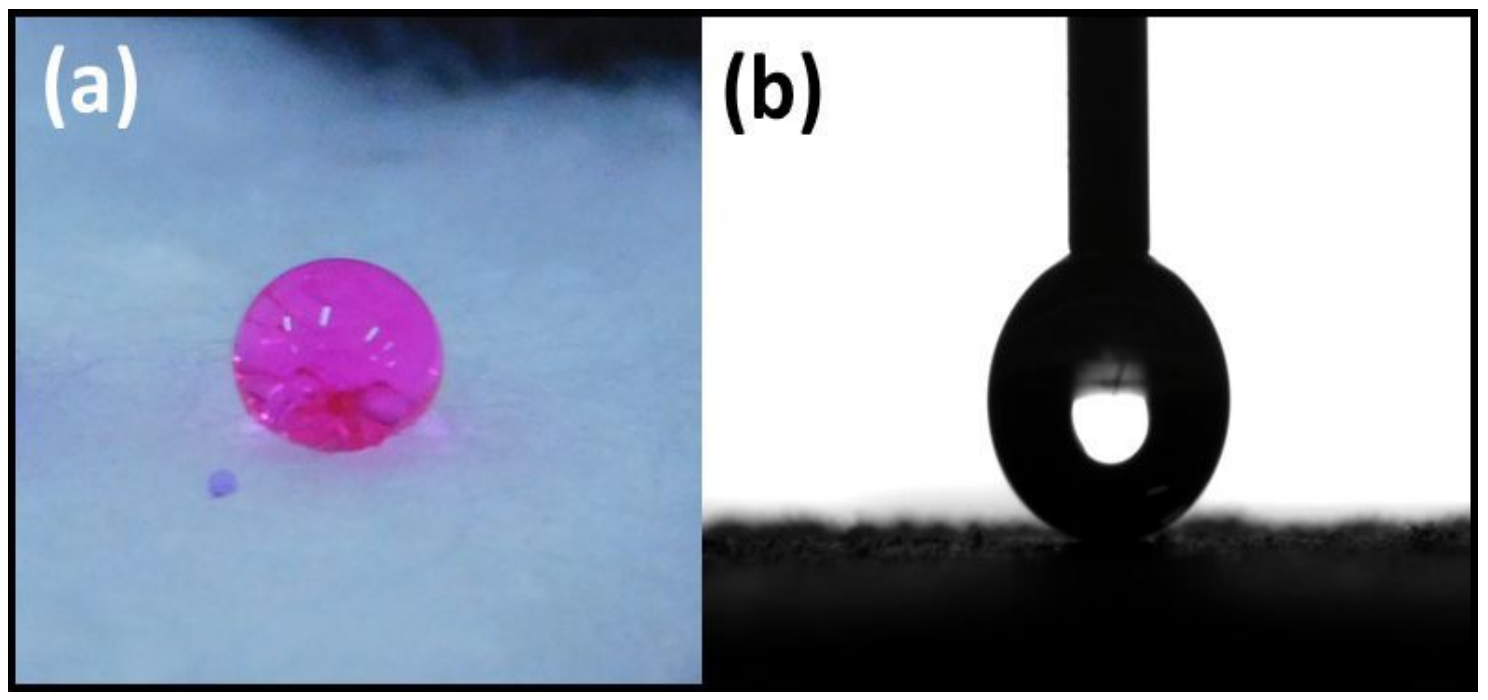

Figure S24. Water droplet (red colour aids visual inspection) suspended on SH-UiO-66@CFs composite: naked eye proof of hydrophobicity (a); Image of a water droplet slowly cast on the hydrophobic surface of the SH-UiO-66@CFs composite with a contact angle of about $163^{\circ}$. 


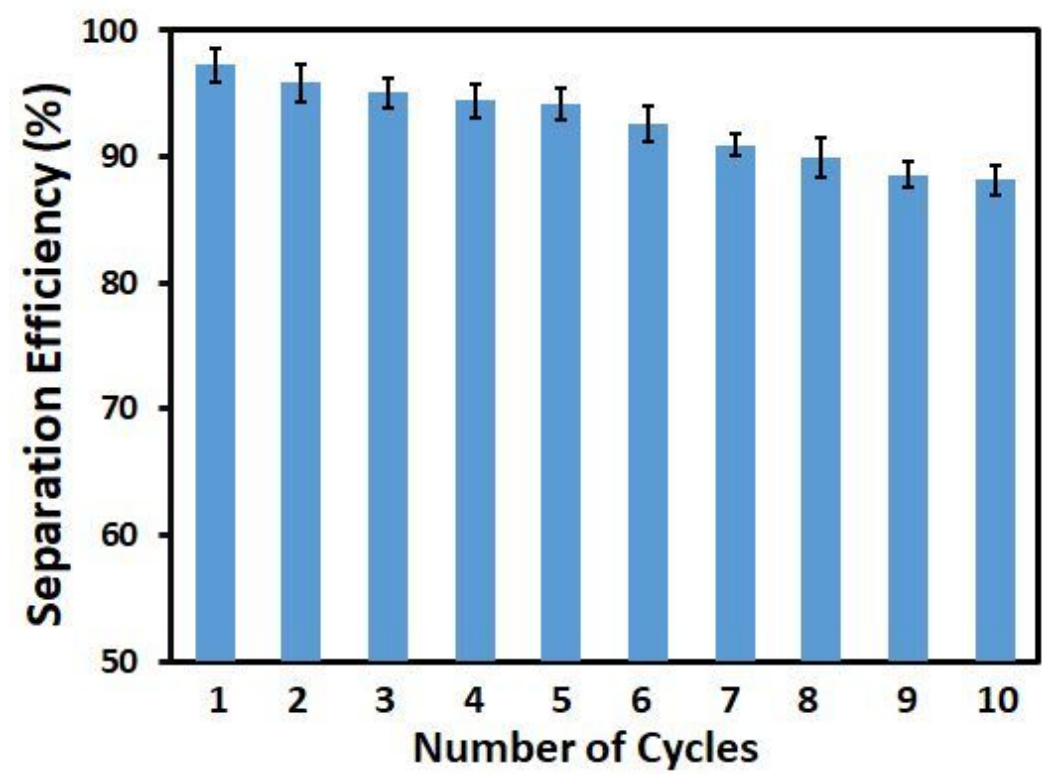

Figure S25. Reusability of SH-UiO-66@CFs composite for oil/water separation experiment (model oil: $\mathrm{CHCl}_{3}$ ).

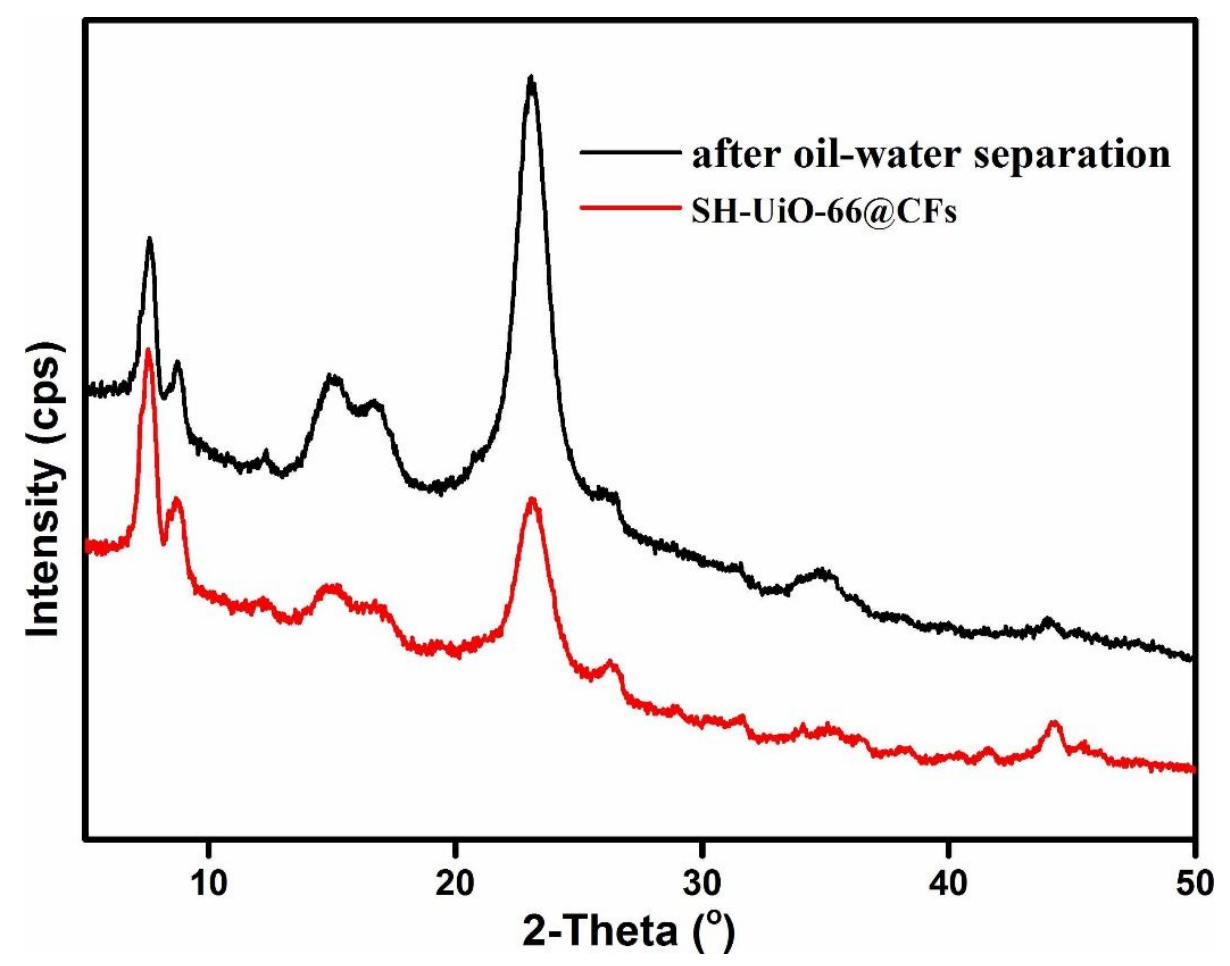

Figure S26. XRPD patterns of SH-UiO-66@CFs composite before (red) and after (black) oilwater separation experiments (model oil: $\mathrm{CHCl}_{3}$ ). 

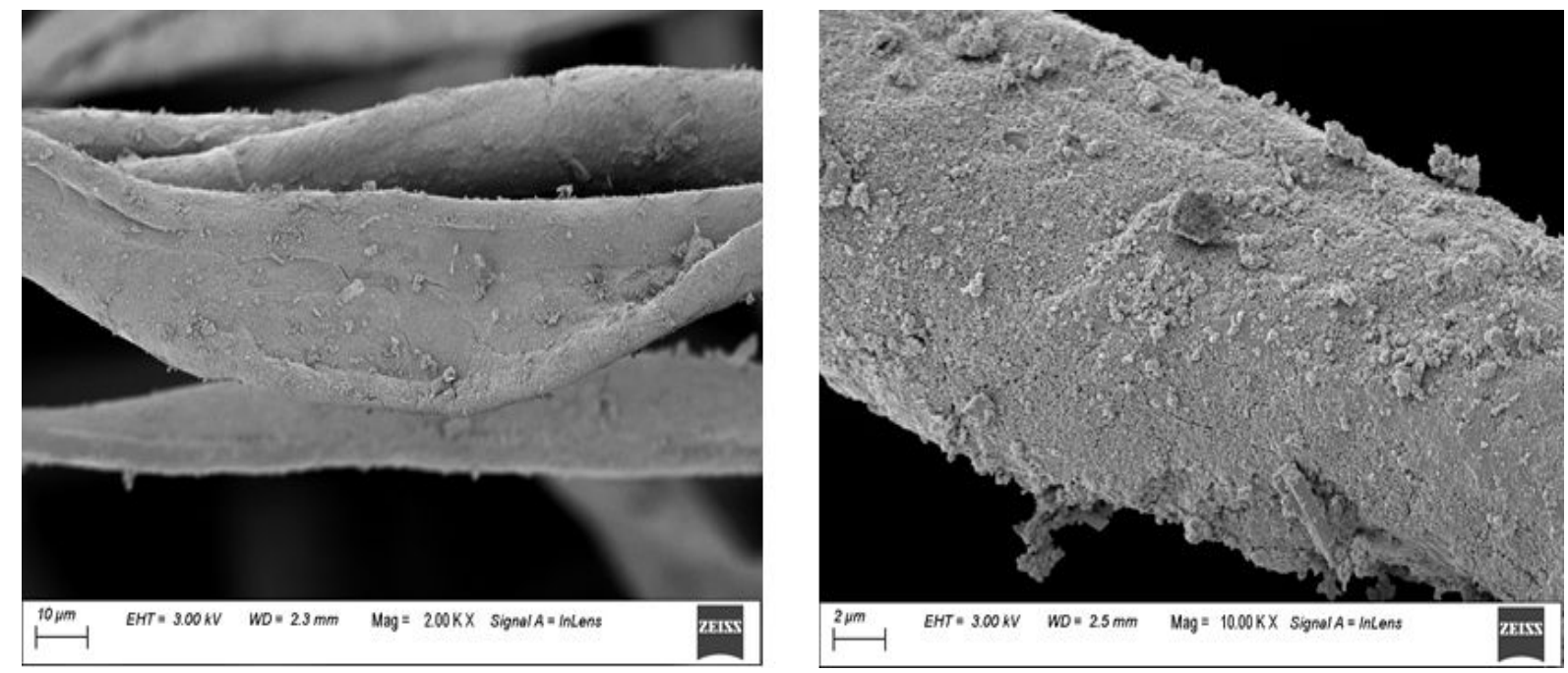

Figure S27. FE-SEM images of reused SH-UiO-66@CFs composite (model oil: $\mathrm{CHCl}_{3}$ ).

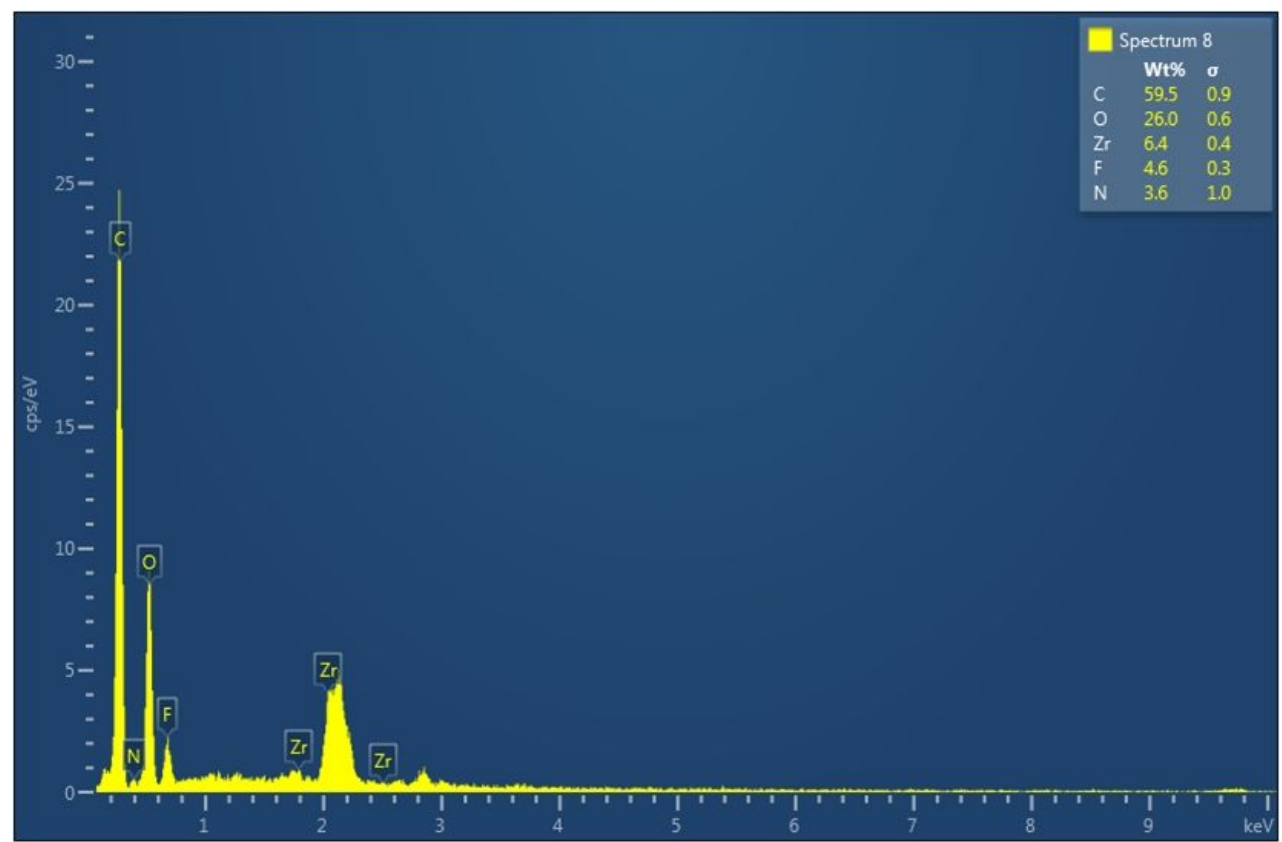

Figure S28. EDX spectrum of reused SH-UiO-66@CFs (model oil: $\mathrm{CHCl}_{3}$ ). 
C Ka1_2
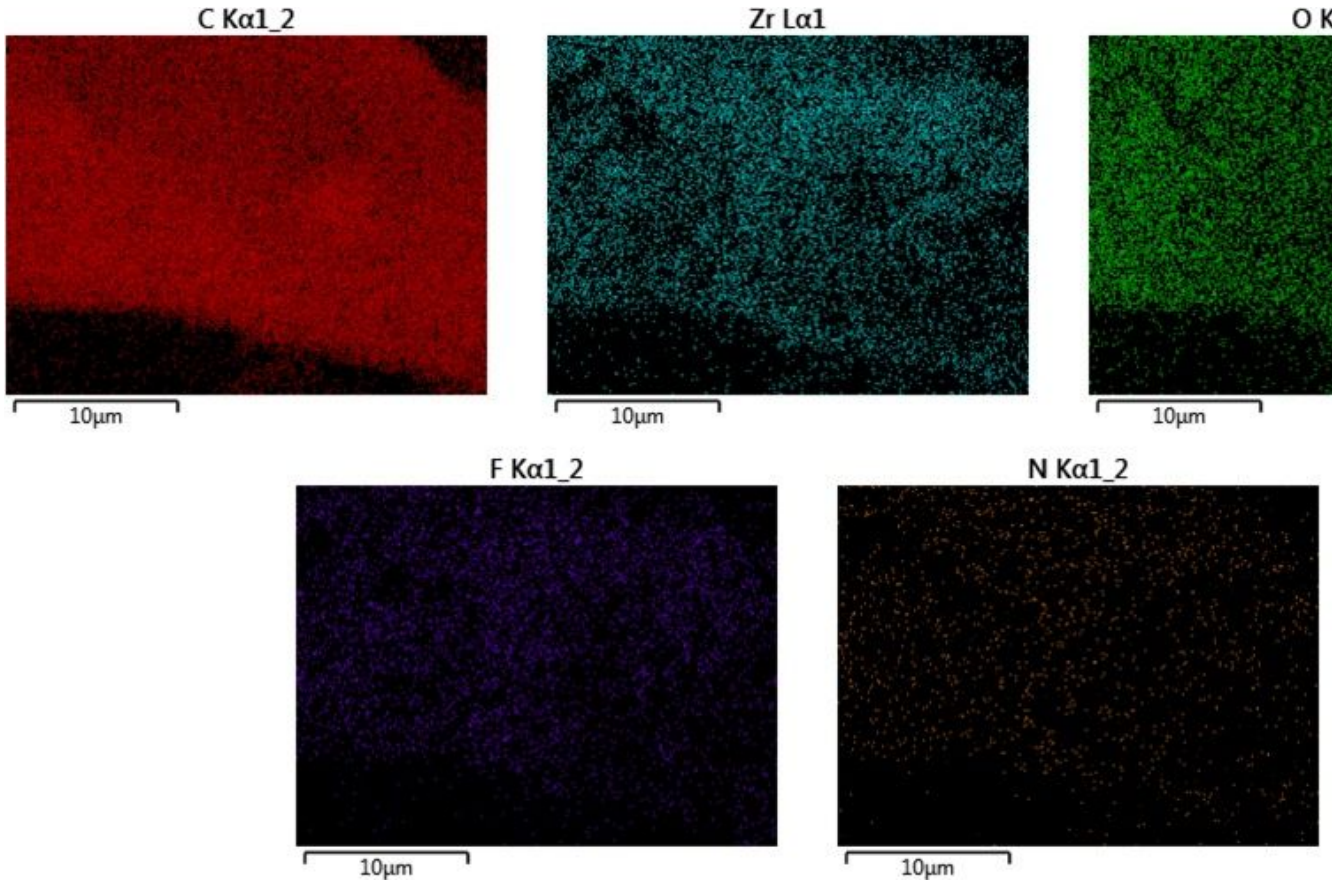

Figure S29. EDX elemental mapping of reused SH-UiO-66@CFs composite (model oil: $\left.\mathrm{CHCl}_{3}\right)$.

Table S1. List of some reported hydrophobic MOFs, summarizing name of MOFs, WCA, constituent ligand, origin of hydrophobicity, investigated form and application.

\begin{tabular}{|c|c|c|c|c|c|c|}
\hline $\begin{array}{l}\text { Sl. } \\
\text { No. }\end{array}$ & MOF & $\begin{array}{l}\text { CA (in } \\
\text { air) } \\
\text { (deg) }\end{array}$ & $\begin{array}{l}\text { Origin of } \\
\text { hydrophobic nature }\end{array}$ & Investigation form & Applications & Ref. \\
\hline 1 & $\begin{array}{l}\text { SH-UiO- } \\
66\end{array}$ & 160 & $\begin{array}{l}\text { 2-trifluoroacetamido } \\
\text { terephthalic acid }\end{array}$ & $\begin{array}{l}\text { MOF coated cotton } \\
\text { fiber }\end{array}$ & $\begin{array}{l}\text { recyclable } \\
\text { oil/water } \\
\text { separation } \\
\text { (gravity driven, } \\
\text { anti-gravity, } \\
\text { under harsh } \\
\text { conditions) and } \\
\text { oil absorption }\end{array}$ & $\begin{array}{l}\text { this } \\
\text { work }\end{array}$ \\
\hline 2 & $\begin{array}{l}\text { PFOA@ } \\
\text { UiO-67 } \\
\& \\
\text { HFBA@ } \\
\text { UiO-66 }\end{array}$ & $\begin{array}{l}147 \& \\
135\end{array}$ & $\begin{array}{l}\text { perfluorooctanoic } \\
\text { acid (PFOA) and } \\
\text { heptafluorobutyric } \\
\text { acid (HFBA) }\end{array}$ & MOF powder & $\begin{array}{l}\text { oil clean up from } \\
\text { aqueous } \\
\text { environment both } \\
\text { under passive } \\
\text { flow (column) } \\
\text { and under } \\
\text { simulated spill } \\
\text { conditions }\end{array}$ & 1 \\
\hline 3 & $\begin{array}{l}\text { UHMOF- } \\
100\end{array}$ & 176 & $\begin{array}{l}\text { bis(trifluoromethyl) } \\
\text { functionalized } \\
\text { aromatic } \\
\text { linker }\end{array}$ & crystalline powder & $\begin{array}{l}\text { oil/water } \\
\text { separation }\end{array}$ & 2 \\
\hline 4 & $\begin{array}{l}\text { ZIF- } \\
\text { 8@rGO@ } \\
\text { Sponge }\end{array}$ & 171 & $\begin{array}{l}\text { micro/nano- } \\
\text { hierarchical } \\
\text { architecture }\end{array}$ & $\begin{array}{l}\text { composite sponge } \\
\text { surface }\end{array}$ & $\begin{array}{l}\text { recyclable } \\
\text { oil/water } \\
\text { separation }\end{array}$ & 3 \\
\hline
\end{tabular}




\begin{tabular}{|c|c|c|c|c|c|c|}
\hline & & & $\begin{array}{l}\text { comprising wrinkled, } \\
\text { reduced rGO nano } \\
\text { sheets intercalated } \\
\text { with ZIF-8 } \\
\text { nanoparticles }\end{array}$ & & & \\
\hline 5 & NMOF-1 & $\begin{array}{l}160- \\
162 \\
171\end{array}$ & $\mathrm{H}_{2} \mathrm{OPE}-\mathrm{C}_{18}$ & $\begin{array}{l}\text { coated glass } \\
\text { substrate }\end{array}$ & self cleaning & $4-5$ \\
\hline 6 & $\begin{array}{l}\text { HFGO@ } \\
\text { ZIF-8 } \\
\text { composite }\end{array}$ & 162 & $\begin{array}{l}\text { highly fluorinated } \\
\text { graphene oxide } \\
\text { (HFGO) }\end{array}$ & $\begin{array}{l}\text { composite material } \\
\text { pellet }\end{array}$ & $\begin{array}{l}\text { oil/ water } \\
\text { separation }\end{array}$ & 6 \\
\hline 7 & $\begin{array}{l}\text { OPA- } \\
\text { UiO-66 \& } \\
\text { OPA- } \\
\text { UiO-66- } \\
\mathrm{SO}_{3} \mathrm{H} \\
\& \\
\text { OPA- } \\
\text { PCN-222 }\end{array}$ & $\begin{array}{l}160 \\
\& \\
162 \\
\& \\
157\end{array}$ & $\begin{array}{l}n \text {-octa- } \\
\text { decylphosphonic } \\
\text { acid (OPA) }\end{array}$ & powder & $\begin{array}{l}\text { high organic } \\
\text { solvent absorption } \\
\text { capacity; } \\
\text { oil/water } \\
\text { separation }\end{array}$ & 7 \\
\hline 8 & PESD-1 & $\begin{array}{l}152 \\
>150\end{array}$ & $\mathrm{H}_{3} \mathrm{BTMB}$ & $\begin{array}{l}\text { single crystal, } \\
\text { powder }\end{array}$ & $\begin{array}{l}\text { selective removal } \\
\text { of organic solvent } \\
\text { from water, } \\
\text { benzene/ } \\
\text { cyclohexane } \\
\text { selective } \\
\text { vapor sorption }\end{array}$ & 8 \\
\hline 9 & $\begin{array}{l}\text { fluorinate } \\
\text { d ZIF-90 }\end{array}$ & 152.4 & $\begin{array}{l}\text { pentalfluorobenzyla- } \\
\text { mine }\end{array}$ & crystal & $\begin{array}{l}\text { recyclable bio- } \\
\text { alcohol recovery } \\
\text { performance from } \\
\text { water }\end{array}$ & 9 \\
\hline 10 & MOFF-2 & $151 \pm 1$ & $\begin{array}{l}\text { fluorinated biphenyl- } \\
\text { based ligands }\end{array}$ & pressed crystal & $\mathrm{N} / \mathrm{A}$ & 10 \\
\hline 11 & $\begin{array}{l}\text { SIM- } \\
2\left(\mathrm{C}_{12}\right) / \mathrm{Al}_{2} \\
\mathrm{O}_{3}\end{array}$ & $>150$ & alkyl chain $\left(\mathrm{C}_{12}\right)$ & $\begin{array}{l}\text { film on } \mathrm{Al}_{2} \mathrm{O}_{3} \\
\text { support }\end{array}$ & $\begin{array}{l}\text { catalyst for } \\
\text { Knoevenagel } \\
\text { condensation and } \\
\mathrm{CO}_{2} / \mathrm{N}_{2} \text { separation } \\
\text { under humid } \\
\text { conditions. }\end{array}$ & 11 \\
\hline 12 & $\begin{array}{l}\text { MIL- } \\
\text { 53(Al)- } \\
\text { AM6 } \\
\text { MIL- } \\
\text { 53(Al)- } \\
\text { AM4 } \\
\text { (AMn: } \\
\text { Amide } \\
\text { with } n- \\
\text { carbon } \\
\text { chain) }\end{array}$ & $>150$ & $\begin{array}{l}\text { hydrophobic alkyl } \\
\text { chains }\end{array}$ & powder & $\mathrm{N} / \mathrm{A}$ & 12 \\
\hline 13 & FMOF-1 & 158 & $\begin{array}{l}3,5- \\
\text { bis(trifluoromethyl)- } \\
1,2,4 \text {-triazolate }\end{array}$ & pellet & $\begin{array}{l}\mathrm{CO}_{2} \text { selective gas } \\
\text { sorption }\end{array}$ & 13 \\
\hline 14 & $\begin{array}{l}\text { PESD-1 } \\
\text { PESD-2 }\end{array}$ & $\begin{array}{l}155.5 \\
159.3 \\
\end{array}$ & $\mathrm{H}_{3} \mathrm{BTMB}$ & powder & $\begin{array}{l}\text { aromatic solvent } \\
\text { adsorption; }\end{array}$ & 14 \\
\hline
\end{tabular}




\begin{tabular}{|l|l|l|l|l|l|l|}
\hline & PESD-3 & 160.8 & & $\begin{array}{l}\text { oil/water } \\
\text { separation }\end{array}$ & \\
\hline
\end{tabular}

Table S2. Contact angle (CA) of SH-UiO-66@CFs after treatment under various conditions.

\begin{tabular}{|l|l|c|}
\hline $\begin{array}{l}\text { SI. } \\
\text { No. }\end{array}$ & \multicolumn{1}{|c|}{ Treatment Conitions } & CA (static) \\
\hline 1 & In dichloromethane $(24 \mathrm{~h})$ & $163^{\circ}$ \\
\hline 2 & In chloroform $(24 \mathrm{~h})$ & $151^{\circ}$ \\
\hline 3 & In carbon tetrachloride $(24 \mathrm{~h})$ & $140^{\circ}$ \\
\hline 4 & In ethyl acetate $(24 \mathrm{~h})$ & $154^{\circ}$ \\
\hline 5 & In hexane $(24 \mathrm{~h})$ & $150^{\circ}$ \\
\hline 6 & In toluene $(24 \mathrm{~h})$ & $151^{\circ}$ \\
\hline 7 & In tap water $(24 \mathrm{~h})$ & $154^{\circ}$ \\
\hline 8 & In artificial sea water $(24 \mathrm{~h})$ & $150^{\circ}$ \\
\hline 9 & In river water $(24 \mathrm{~h})$ & $151^{\circ}$ \\
\hline 10 & In lake water $(24 \mathrm{~h})$ & $152^{\circ}$ \\
\hline 11 & In pH $=2(24 \mathrm{~h})$ & $152^{\circ}$ \\
\hline 12 & In pH $=12(24 \mathrm{~h})$ & $148^{\circ}$ \\
\hline 13 & In UV exposure $(4$ days $)$ & $155^{\circ}$ \\
\hline 14 & In ice water $(24 \mathrm{~h})$ & $143^{\circ}$ \\
\hline 14 & In boiling water $(24 \mathrm{~h})$ & $0^{\circ}$ \\
\hline
\end{tabular}

Table S3. Density of oils used for oil/water separation experiments.

\begin{tabular}{|l|l|c|}
\hline $\begin{array}{l}\text { Sl. } \\
\text { No. }\end{array}$ & Oils & Density $\left(\mathbf{g ~ c m}^{-3}\right)$ \\
\hline 1 & dichloromethane & 1.33 \\
\hline 2 & chloroform & 1.49 \\
\hline 3 & carbon tetrachloride & 1.59 \\
\hline 4 & ethyl acetate & 0.90 \\
\hline 5 & hexane & 0.65 \\
\hline 6 & toluene & 0.86 \\
\hline 7 & motor oil & 0.87 \\
\hline 8 & silicone oil & 0.97 \\
\hline 9 & gasoline & 0.78 \\
\hline 10 & kerosene & 0.81 \\
\hline
\end{tabular}

Table S4. Comparative absorption capacities of various absorbents for oil absorption.

\begin{tabular}{|l|l|l|l|l|}
\hline $\begin{array}{l}\text { Sl. } \\
\text { No. }\end{array}$ & Absorbents & Type of Oil & $\begin{array}{l}\text { Absorption Capacity } \\
(\mathbf{g} / \mathbf{g})\end{array}$ & Ref. \\
\hline 1 & SH-UiO-66@CFs & motor oil & 45.23 & this \\
& & silicone oil & 38.06 & work \\
& & gasoline & 31.87 & \\
\hline 2 & cotton fiber modified & kerosene & 29.41 & 15 \\
\hline
\end{tabular}




\begin{tabular}{|c|c|c|c|c|}
\hline & via the sol-gel method & $\begin{array}{l}\text { lubrication oil } \\
\text { crude oil }\end{array}$ & $\begin{array}{l}44.24 \\
57.01\end{array}$ & \\
\hline 3 & $\begin{array}{l}\text { modified jute fiber } \\
\text { via the sol-gel method }\end{array}$ & $\begin{array}{l}\text { crude oil } \\
\text { diesel oil } \\
\text { lubrication oil }\end{array}$ & $\begin{array}{l}7.41 \\
8.48 \\
10.29\end{array}$ & 16 \\
\hline 4 & mesoporous silica aerogel & $\begin{array}{l}\text { petrol oil } \\
\text { diesel oil }\end{array}$ & $\begin{array}{l}19.1 \\
18.6\end{array}$ & 17 \\
\hline 5 & ultralight cellulose-based aerogel & $\begin{array}{l}\text { pump oil } \\
\text { diesel oil }\end{array}$ & $\begin{array}{l}22.60 \\
20.9\end{array}$ & 18 \\
\hline 6 & $\begin{array}{l}\text { i) cotton modified using } \\
\mathrm{P}-S i O_{2} \text { nanoparticles } \\
\text { ii) } \mathrm{Kapok} \text { modified using } \\
\mathrm{P}_{-} \mathrm{SiO}_{2} \text { nanoparticles }\end{array}$ & $\begin{array}{l}\text { i) diesel oil } \\
\text { ii) diesel oil }\end{array}$ & $\begin{array}{l}\text { i) } 20 \\
\text { ii) } 23\end{array}$ & 19 \\
\hline 7 & $\mathrm{PDMS} \mathrm{TiO}_{2}-\mathrm{PU}$ sponge & diesel oil & 14.20 & 20 \\
\hline 8 & cellulose-based aerogels & $\begin{array}{l}\text { crude oil } \\
\text { diesel oil } \\
\text { lubrication oil } \\
\text { silicone oil }\end{array}$ & $\begin{array}{l}77.08 \\
91.82 \\
105.83 \\
89.72\end{array}$ & 21 \\
\hline 9 & $\begin{array}{l}\text { superhydrophobic/superoleophilic } \\
\text { sawdust }\end{array}$ & crude oil & 17.50 & 22 \\
\hline
\end{tabular}

\section{References:}

1. DeChellis, D. M.; Ngule, C. M.; Genna, D. T., Removal of hydrocarbon contaminants from water with perfluorocarboxylated UiO-6X derivatives. J. Mater. Chem. A 2020, 8, 58485852.

2. Mukherjee, S.; Kansara, A. M.; Saha, D.; Gonnade, R.; Mullangi, D.; Manna, B.; Desai, A. V.; Thorat, S. H.; Singh, P. S.; Mukherjee, A.; Ghosh, S. K., An ultrahydrophobic fluorous metal-organic framework derived recyclable composite as a promising platform to tackle marine oil spills. Chem. - Eur. J. 2016, 22, 10937-10943.

3. Gu, J.; Fan, H.; Li, C.; Caro, J.; Meng, H., Back Cover: Robust superhydrophobic/superoleophilic wrinkled microspherical MOF@rGO composites for efficient oil-water separation. Angew. Chem., Int. Ed. 2019, 58, 5464-5464.

4. Roy, S.; Suresh, V. M.; Maji, T. K., Self-cleaning MOF: realization of extreme water repellence in coordination driven self-assembled nanostructures. Chem. Sci. 2016, 7, 22512256.

5. Samanta, D.; Roy, S.; Sasmal, R.; Saha, N. D.; Pradeep, K. R.; Ranjani, V.; Agasti, S. S.; Maji, T. K., Solvent adaptive dynamic metal-organic soft hybrid for imaging and biological delivery. Angew. Chem., Int. Ed. 2019, 131, 5062-5066. 
6. Jayaramulu, K.; Datta, K. K. R.; Rösler, C.; Petr, M.; Otyepka, M.; Zboril, R.; Fischer, R. A., Biomimetic superhydrophobic/superoleophilic highly fluorinated graphene oxide and ZIF-8 composites for oil-water separation. Angew. Chem., Int. Ed. 2016, 55, 1178-1182.

7. Sun, Y.; Sun, Q.; Huang, H.; Aguila, B.; Niu, Z.; Perman, J. A.; Ma, S., A molecularlevel superhydrophobic external surface to improve the stability of metal-organic frameworks. J. Mater. Chem. A 2017, 5, 18770-18776.

8. Rao, K. P.; Higuchi, M.; Sumida, K.; Furukawa, S.; Duan, J.; Kitagawa, S., Design of Superhydrophobic Porous Coordination Polymers through the Introduction of External Surface Corrugation by the Use of an Aromatic Hydrocarbon Building Unit. Angew. Chem., Int. Ed. 2014, 53, 8225-8230.

9. Liu, C.; Liu, Q.; Huang, A., A superhydrophobic zeolitic imidazolate framework (ZIF90) with high steam stability for efficient recovery of bioalcohols. Chem. Commun. 2016, 52, 3400-3402.

10. Chen, T.-H.; Popov, I.; Zenasni, O.; Daugulis, O.; Miljanic, O. Š., Superhydrophobic perfluorinated metal-organic frameworks. Chem. Commun. 2013, 49, 6846-6848.

11. Aguado, S.; Canivet, J.; Farrusseng, D., Engineering structured MOF at nano and macroscales for catalysis and separation. J. Mater. Chem. 2011, 21, 7582-7588.

12. Nguyen, J. G.; S. M. Cohen, moisture-resistant and superhydrophobic metal-organic frameworks obtained via postsynthetic modification. J. Am. Chem. Soc. 2010, 132, 4560-4561. 13. Moghadam, P. Z.; Ivy, J. F.; Arvapally, R. K.; Santos, A. M. d.; Pearson, J. C.; Zhang, L.; Tylianakis, E.; Ghosh, P.; Oswald, I. W. H.; Kaipa, U.; Wang, X.; Wilson, A. K.; Snurr, R. Q.; Omary, M. A., Adsorption and molecular siting of $\mathrm{CO}_{2}$, water, and other gases in the superhydrophobic, flexible pores of FMOF-1 from experiment and simulation. Chem. Sci. 2017, 8, 3989-4000.

14. Rao, K. P.; Higuchi, M.; Suryachandram, J.; Kitagawa, S., Temperature-stable compelled composite superhydrophobic porous coordination polymers achieved via an unattainable de novo synthetic method. J. Am. Chem. Soc. 2018, 140, 13786-13792.

15. Lv, N.; Wang, X.; Peng, S.; Luo, L.; Zhou, R., Superhydrophobic/superoleophilic cotton-oil absorbent: preparation and its application in oil/ water separation. RSC Adv. 2018, $8,30257-30264$.

16. Lv, N.; Wang, X.; Peng, S.; Zhang, H.; Luo, L., Study of the kinetics and equilibrium of the adsorption of oils onto hydrophobic jute fiber modified via the sol-gel method. Int. J. Environ. Res. Public Health 2018, 15, 969. 
17. Zhang, C.; Dai, C.; Zhang, H.; Peng, S.; Wei, X.; Hu, Y., Regeneration of mesoporous silica aerogel for hydrocarbon adsorption and recovery. Mar. Pollut. Bull. 2017, 122, 129-138. 18. Zhang, H.; Li, Y.; Xu, Y.; Lu, Z.; Chen, L.; Huang, L.; Fan, M., Versatile fabrication of a superhydrophobic and ultralight cellulose-based aerogel for oil spillage clean-up. Phys. Chem. Chem. Phys. 2016, 18, 28297-28306.

19. Lee, J. H.; Kim, D. H.; Han, S. W.; Kim, B. R.; Park, E. J.; Jeong, M.-G.; Kim, J. H.; Kim, Y. D., Fabrication of superhydrophobic fibre and its application to selective oil spill removal. Chem. Eng. J. 2016, 289, 1-6.

20. Shuai, Q.; Yang, X.; Luo, Y.; Tang, H.; Luo, X.; Tan, Y.; Ma, M., A superhydrophobic poly(dimethylsiloxane) $-\mathrm{TiO}_{2}$ coated polyurethane sponge for selective absorption of oil from water. Mater. Chem. Phys. 2015, 162, 94-99.

21. Yin, T.; Zhang, X.; Liu, X.; Wang, C., Resource recovery of Eichhornia crassipes as oil superabsorbent. Mar. Pollut. Bull. 2017, 118, 267-274.

22. Zang, D.; Liu, F.; Zhang, M.; Gao, Z.; Wang, C., Novel superhydrophobic and superoleophilic sawdust as a selective oil sorbent for oil spill cleanup. Chem. Eng. Res. Des. 2015, 102, 34-41. 